\title{
Intrinsic Mechanisms for Adaptive Gain Rescaling in Barrel Cortex
}

\author{
Marta Díaz-Quesada and Miguel Maravall \\ Instituto de Neurociencias de Alicante, Universidad Miguel Hernández de Elche-Consejo Superior de Investigaciones Científicas, Campus de San Juan, \\ 03550 Sant Joan d'Alacant, Spain
}

\begin{abstract}
Barrel cortex neuronal responses adapt to changes in the statistics of complex whisker stimuli. This form of adaptation involves an adjustment in the input-output tuning functions of the neurons, such that their gain rescales depending on the range of the current stimulus distribution. Similar phenomena have been observed in other sensory systems, suggesting that adaptive adjustment of responses to ongoing stimulus statistics is an important principle of sensory function. In other systems, adaptation and gain rescaling can depend on intrinsic properties; however, in barrel cortex, whether intrinsic mechanisms can contribute to adaptation to stimulus statistics is unknown. To examine this, we performed whole-cell patch-clamp recordings of pyramidal cells in acute slices while injecting stochastic current stimuli. We induced changes in statistical context by switching across stimulus distributions. The firing rates of neurons adapted in response to changes in stimulus statistics. Adaptation depended on the form of the changes in stimulus distribution: in vivo-like adaptation occurred only for rectified stimuli that maintained neurons in a persistent state of net depolarization. Under these conditions, neurons rescaled the gain of their input-output functions according to the scale of the stimulus distribution, as observed in vivo. This stimulus-specific adaptation was caused by intrinsic properties and correlated strongly with the amplitude of calciumdependent slow afterhyperpolarizations. Our results suggest that widely expressed intrinsic mechanisms participate in barrel cortex adaptation but that their recruitment is highly stimulus specific.
\end{abstract}

Key words: vibrissa; somatosensory; noise; sensory coding; slice; afterhyperpolarization; gain control

\section{Introduction}

Adaptation, the accommodation of neuronal responses to ongoing stimulation, occurs across species and sensory modalities. Often, adaptation reflects adjustments of neuronal stimulus-response relationships to the stimulus statistics characteristic of the current sensory environment: such adjustments can result in an optimization of information transmission (Shapley et al., 1972; Shapley and Victor, 1978, 1979; Smirnakis et al., 1997; Wainwright, 1999; Brenner et al., 2000; Fairhall et al., 2001; Dean et al., 2005; Sharpee et al., 2006). Adaptation can occur to changes in different stimulus statistical properties, including mean, variance, and correlations (Smirnakis et al., 1997; Muller et al., 1999; Kvale and Schreiner, 2004; Dean et al., 2005; Hosoya et al., 2005; Bonin et al., 2006; Nagel and Doupe, 2006; Sharpee et al., 2006) (for review, see Wark et al., 2007).

Received July 11, 2007; accepted Nov. 29, 2007.

This work was supported by Human Frontier Science Program Grant RG0043/2004-C, the European Commission Marie Curie Grant MIRG-2004-511273, and the Spanish Ministry of Education and Science Grant BFU2006-04791/ $\mathrm{BFI}$, cofunded by the European Regional Development Fund. M.M. is a Ramón y Cajal Research Fellow (Spanish Ministry of Education and Science). M.D.-Q. is an I3P predoctoral researcher (Consejo Superior de Investigaciones (ientificas). We thank T. Oertner for advice on the data acquisition setup and for Matlab code, K. Svoboda and R. Petersen for Matlab code, J. Bacelo for help on the initial setup, and A. Alenda, M. Diamond, L. Martinez, and R. Petersen for comments on this project and manuscript.

Correspondence should be addressed to Miguel Maravall, Instituto de Neurociencias de Alicante, Universidad Miguel Hernández de Elche-Consejo Superior de Investigaciones Científicas, Campus de San Juan, Apartado 18, 03550 Sant Joan d'Alacant, Spain. E-mail: mmaravall@umh.es.

DOI:10.1523/JNEUROSCI.4931-07.2008

Copyright $\odot 2008$ Society for Neuroscience $\quad$ 0270-6474/08/280696-15\$15.00/0
In the barrel cortex, it is established that responses to repetitive stimulation accommodate, a phenomenon hypothesized to play a role in tactile representations (Simons, 1978; Ahissar et al., 2000, 2001; Castro-Alamancos and Oldford, 2002; Chung et al., 2002; Garabedian et al., 2003; Khatri et al., 2004; Webber and Stanley, 2004, 2006). As in other systems, adaptation is stimulus specific (Derdikman et al., 2006; Katz et al., 2006). Adaptation to stimulus statistics is also present in barrel cortex (Garcia-Lazaro et al., 2007; Maravall et al., 2007). Neurons adjust their coding properties by rescaling their input-output functions in proportion to the stimulus distribution, a rescaling that maintains the information that spikes convey about stimulus features (Maravall et al., 2007).

Intrinsic properties can contribute strongly to adaptation. For example, in primary visual cortex, slow contrast adaptation relies on a sodium-dependent slow afterhyperpolarization (sAHP) (Carandini and Ferster, 1997; Sanchez-Vives et al., 2000a,b); in salamander retina, calcium-dependent mechanisms and sodium current inactivation underlie changes in gain during contrast adaptation in different cell types (Kim and Rieke, 2001, 2003; Rieke, 2001). Different mechanisms, underlying contrast adaptation in different cell types, can have similar effects (Baccus and Meister, 2002; Demb, 2002). Moreover, modeling studies have shown that certain forms of adaptation can be generic, appearing even in simplified models that capture very general features of response generation. Gain modulation in these models arises from interactions between spike generation nonlinearities and the scale of 
the stimulus distribution (Rudd and Brown, 1997; Aguera y Arcas and Fairhall, 2003; Paninski et al., 2003; Borst et al., 2005; Yu et al., 2005; Gaudry and Reinagel, 2007). These findings underscore that, for any instance of adaptation, it is important to understand whether it is generic or dependent on particular neuronal properties and whether it is common across stimulus conditions or stimulus specific (Wark et al., 2007).

In barrel cortex, adaptation to repetitive stimulation is attributable primarily to synaptic mechanisms (Castro-Alamancos and Oldford, 2002; Chung et al., 2002; Katz et al., 2006). Whether intrinsic mechanisms could contribute to adaptation to changes in stimulus statistics has been unknown. Using slice recordings, here we show that intrinsic mechanisms suffice to produce in vivo-like, stimulus-specific adaptation.

\section{Materials and Methods}

All procedures complied with Society for Neuroscience, European Community, and Spanish policies for the care and use of animals in research.

\section{Slice preparation}

We prepared acute cortical slices from Wistar rats at age 12-20 d postnatal. Slice preparation followed standard procedures. Slices $(300 \mu \mathrm{m}$ thick) were cut on an Integraslice 7550MM microtome (Campden Instruments, Loughborough, UK) with the brain submerged in a chilled $\left(2-5^{\circ} \mathrm{C}\right)$ cutting solution bubbled with carbogen $\left(95 \% \mathrm{O}_{2}-5 \% \mathrm{CO}_{2}\right)$ and glued to a custom-made block to standardize cutting angle. The solution contained the following (in mM): 110 choline chloride, $25 \mathrm{NaHCO}_{3}, 25$ D-glucose, $11.6 \mathrm{Na}$-ascorbate, $7 \mathrm{MgSO}_{4}, 3.1 \mathrm{Na}$-pyruvate, $2.5 \mathrm{KCl}, 1.25$ $\mathrm{NaH}_{2} \mathrm{PO}$, and $0.5 \mathrm{CaCl}_{2}$. After cutting, slices were transferred to a submerged holding chamber containing artificial CSF (ACSF), incubated at $35^{\circ} \mathrm{C}$ for $\sim 30 \mathrm{~min}$, and then kept at room temperature until used. The composition of the normal ACSF was the following (in mM): $127 \mathrm{NaCl}$, $25 \mathrm{NaHCO}_{3}, 25$ D-glucose, $2.5 \mathrm{KCl}, 2 \mathrm{CaCl}_{2}, 1 \mathrm{MgCl}_{2}$, and 1.25 $\mathrm{NaH}_{2} \mathrm{PO}_{4}$, pH 7.3 (303 mOsm). All chemicals were from Sigma-Aldrich (Madrid, Spain) unless otherwise noted. Drugs, including the synaptic blockers ( $R S$ )-3-(2-carboxypiperazin-4-yl)-propyl-1-phosphonic acid (RS-CPP) and 6-cyano-7-nitroquinoxaline-2,3-dione (CNQX) (both from Tocris Bioscience, Bristol, UK), the calcium influx blocker $\mathrm{CdCl}_{2}$, and the serotonergic $\left(5-\mathrm{HT}_{2}\right)$ agonist $\alpha$-methyl-serotonin maleate salt $(\alpha \mathrm{me} 5 \mathrm{HT})$, were added to the bath perfusion as needed.

\section{Stimulation and data acquisition}

Whole-cell somatic patch-clamp recordings were performed according to standard procedures. Using infrared differential interference contrast optics to visualize neurons and barrels, we restricted our search to pyramidal neurons contained within barrel columns. Cells were selected according to morphology and layer position. Recordings were performed at room temperature or at $34 \pm 1^{\circ} \mathrm{C}$, as detailed in Results. Patch electrodes were pulled from borosilicate glass $[1.5 \mathrm{~mm}$ external diameter, $0.86 \mathrm{~mm}$ internal diameter (World Precision Instruments, Stevenage, $\mathrm{UK}) ; 3-6 \mathrm{M} \Omega$ ] and filled with potassium-based internal solution. Solution contained the following (in $\mathrm{mm}$ ): either $130 \mathrm{~K}$-methylsulfonate or K-methylsulfate, $10 \mathrm{Na}$-phosphocreatine, 10 HEPES, $4 \mathrm{MgCl}_{2}, 4 \mathrm{Na}_{2}$ ATP, $3 \mathrm{Na}$-ascorbate, and $0.4 \mathrm{Na}_{2}$-GTP; or $120 \mathrm{~K}$-gluconate, $15 \mathrm{Na}$ phosphocreatine, 10 HEPES, $5 \mathrm{NaCl}, 2 \mathrm{MgCl}_{2}, 4 \mathrm{Mg}_{2}$-ATP, $3 \mathrm{Na}$ ascorbate, $1.1 \mathrm{EGTA}, 0.4 \mathrm{Na}_{2}-\mathrm{GTP}$, and $0.1 \mathrm{CaCl}_{2}$; adjusted to $\mathrm{pH} 7.33$ (287-303 mOsm, slightly hypo-osmotic compared with the external ACSF). Recordings were not corrected for liquid junction potential. Neurons were monitored for access resistance $(<20 \mathrm{M} \Omega)$ as well as for changes in input and access resistances, in spike height $(<5 \%)$, and in resting membrane potential. Recordings were discarded if they did not remain stable according to these criteria over a minimum of $1-2 \mathrm{~h}$, the time necessary to collect enough spikes. During break-in, each neuron was tested with a series of pulses to check whether it responded with the "regular spiking" phenotype characteristic of many excitatory cortical neurons (McCormick et al., 1985); those that did not were excluded from the analyzed dataset.

Data were acquired with an Axon Multiclamp 700-B amplifier (Mo- lecular Devices, Union City, CA), filtered at $10 \mathrm{kHz}$, and sampled at 20 $\mathrm{kHz}$ with an analog-to-digital board (PCI 6040-E; National Instruments, Austin, TX) controlled with software custom-written in Matlab (MathWorks, Natick, MA) (code written by T. Pologruto, B. L. Sabatini, K. Svoboda, T. G. Oertner, and M. Maravall).

\section{Stimulus design}

Current stimuli were generated in Matlab and injected via the recording electrode. Stimulus values changed over two different timescales. First, stochastic stimulus waveforms were generated as white noise sampled from a Gaussian distribution and filtered by convolution with a Gaussian waveform with SD of $2 \mathrm{~ms}$. Individual current values therefore fluctuated rapidly, over a timescale of a few milliseconds. Second, stimuli had a variance and/or mean that switched cyclically over a timescale of seconds (full cycle duration was 5 s, i.e., each high or low variance "epoch" lasted $2.5 \mathrm{~s}$ ) (see Fig. 1A) (for details of the different switching protocols, see Results). Each switch was smoothed over $10 \mathrm{~ms}$. Therefore, the statistical distribution from which stimulus values were drawn, which represented the context within which stimuli arrived, changed every few seconds. Acquisition was broken into $32 \mathrm{~s}$ trials, and stimuli were $30 \mathrm{~s}$ long; the extra recording time at the end of each trial allowed measurement of poststimulus changes in membrane potential.

Stimuli received by barrel cortex neurons during sensory experience in vivo consist of extended sequences of excitatory and inhibitory synaptic events arriving at irregular, rapidly fluctuating intervals. Our choice of white noise stimulus protocols was motivated by several advantages. First, white noise protocols provide a useful way to create extended, continuous stimuli with rapid variations and parametric changes in mean and variance, allowing for a standardized experimental design for comparative study across neurons. Second, white noise stimuli present a large, unbiased sample of input values, permitting spike-triggered analysis; cortical responses to electrical white noise stimulation are well characterized (Mainen and Sejnowski, 1995; Nowak et al., 1997; Tang et al., 1997). Third, cortical neurons in vitro produce responses to white noise currents that share properties of in vivo activity, and Gaussian white noise provides a basic model for the distribution of total current inputs at the soma under conditions of intense synaptic bombardment (Mainen and Sejnowski, 1995; Destexhe et al., 2001; Rauch et al., 2003). Finally, modeling work has shown that, under many circumstances, conductancedriven stimuli produce similar responses to white noise current stimuli: in models that capture key properties of experimentally recorded neurons, the response properties of model neurons driven by conductance stimuli map onto the response properties of equivalent neurons driven by current stimuli (Rauch et al., 2003; Jolivet and Gerstner, 2004; La Camera et al., 2004; Richardson, 2004; Jolivet et al., 2006).

To compare sAHP current magnitude across neurons using a standardized protocol, we delivered pulse trains consisting of 10 square current pulses ( $1 \mathrm{~ms}$ duration) at $100 \mathrm{~Hz}$. Pulse amplitude was set sufficiently high to generate action potentials reliably.

\section{Analysis}

All analyses were performed in Matlab. Spikes were extracted from raw membrane potential traces by thresholding. Each spike time was defined as the time of maximum acceleration of the membrane potential waveform, before the spike peak.

Firing rates. Firing rates were determined by counting spikes over successive time windows (for rate plots, window size was $50 \mathrm{~ms}$ ) and dividing the tally by the duration of the windows. We also calculated rates by taking the inverse of the mean interstimulus interval computed over three or four spikes in each relevant time window; this approach made no qualitative difference to the results, so it is not reported in the final data. Adaptation was assessed by measuring relative changes in firing rate during high- or low-variance epochs. To do so, for low-variance epochs, we computed an "adaptation ratio," defined as the ratio of the rate measured at steady state (time window from 2.3 to $2.5 \mathrm{~s}$ after switching the stimulus distribution) divided by the rate measured immediately after switching (time window from 0 to $100 \mathrm{~ms}$ after switching). With this definition, stronger adaptation corresponded to larger ratios $(>1)$. A similar ratio measure was computed for high-variance periods. Because 
ratios computed for high- and low-variance epochs gave equivalent results, for simplicity we present only the low-variance values.

An adaptation time course was estimated for the rate curve of each neuron (see Fig. $2 B$ ) by focusing on the low-variance part of the curve and finding the first time bin at which the firing rate had recovered from its minimum to within a factor of $1 / e$ times the distance to its steady-state value. For rate curves well fit by a single exponential, this number is equivalent to the decay time constant of the fit, whereas for rate curves with a more complicated structure, it also provides a well defined criterion allowing comparison across experiments.

Stimulus-response relationships: linear-nonlinear representation. We evaluated the neuronal transformation of current into spikes, or neuronal stimulus-response relationship, separately for high- and lowvariance epochs. This allowed us to determine which, if any, elements of the neuronal transformation changed depending on stimulus statistics. To constrain analyses to periods when responses had reached their adapted steady state, we discarded data collected $<1 \mathrm{~s}$ after each variance switch.

Stimulus-response relationships were characterized with a linearnonlinear (LN) representation (Brenner et al., 2000; Chichilnisky, 2001) (for review, see Bialek and De Ruyter Van Steveninck, 2005; Schwartz et al., 2006). Because neurons are sensitive to particular stimulus properties or features, a reasonable way to characterize the function of a neuron is to identify the (hopefully reduced) set of properties relevant to its response and then determine the sensitivity of the response to the presence of those properties in a stimulus. In the LN description, a neuron is represented as a device that, first, linearly filters the stimulus according to the feature selectivity of the neuron, and, second, generates spikes with probability given by a static nonlinear tuning curve, or input-output function, acting on the filtered stimulus. Provided that the information-bearing elements of the response of the neuron are well described by its time-varying instantaneous firing rate, this description can provide a very good picture of the stimulus-response relationship of the neuron. We applied the technique as described previously (Maravall et al., 2007).

Feature selectivity: extraction of linear filters. For each neuron and stimulus condition, we first identified the particular stimulus properties that influenced the response, i.e., the particular features to which the neuron was selective. To do this, we first compared the distribution of stimuli associated with spikes with a random sample of stimuli uncorrelated with spikes, to identify what was different about the stimuli that evoked responses (de Boer and Kuyper, 1968; Bryant and Segundo, 1976; de Ruyter van Steveninck and Bialek, 1988). The spike-triggered distribution was stored as a set of stimulus segments, each of which began $80 \mathrm{~ms}$ before a spike and had $1 \mathrm{~ms}$ resolution. The random distribution comprised segments beginning at random times. Given the sets of spike-triggered and random stimuli, we sought a description of the stimulus properties to which the neuron was selective. The simplest such description is given by averaging the spike-evoking stimulus distribution and comparing this spike-triggered average (STA) with the average of the randomly drawn stimuli. If spike-evoking stimuli differ on average from the original distribution, the STA can represent the most relevant stimulus feature; however, the STA may not provide a good description of feature selectivity (Chichilnisky, 2001; Paninski, 2003). For example, this can happen if a neuron has symmetric stimulus sensitivity, with equal responses to positive and negative values: spikes are then equally likely to be evoked by stimuli of opposite sign, and the spike-triggered average will cancel out (Maravall et al., 2007). Neurons may also be sensitive to more than one stimulus feature (Touryan et al., 2002; Aguera y Arcas et al., 2003; Rust et al., 2005; Slee et al., 2005). Therefore, in addition to extracting the STA [determining whether the spike-triggered and original (prior) stimulus distributions differ on average], it is useful to compute how the distributions differ in shape. This can be done by spike-triggered covariance (STC) analysis, which entails computing the covariance matrix of the spike-triggered distribution and subtracting from it the covariance matrix of the prior distribution (Brenner et al., 2000). The directions in stimulus space along which the distributions are significantly different in shape are those directions along with the variances of the distributions are most different. To find these directions, one first computes the eigenvalues and eigenvectors of the covariance difference matrix, obtained by the subtraction of covariance matrices mentioned above. Then, one identifies the eigenvalues whose absolute magnitude is larger than can be expected from chance. The eigenvectors corresponding to those eigenvalues define the relevant directions in stimulus space and therefore the relevant set of stimulus features, or linear filters, to which the neuron is selective. A detailed description of STC applied to the vibrissa system is given by Maravall et al. (2007).

In summary, we performed STA and STC analysis for each neuron and stimulus condition. We determined both the STA and one to two filters resulting from STC analysis. This set of filters included the stimulus features that most significantly affected the response of the neuron.

Sensitivity: computation of nonlinear tuning curves. After extracting the linear filters as described above, each filter was applied to the input current stimulus over the entire sequence of time points. The resulting sequence of filtered stimulus values represented the stimulus "as seen through" the relevant filter. The value at each time point measured how similar the latest stimulus segment was to the filter. With this sequence as input and the recorded spike history of the neuron as output, we could now map the relationship between filtered stimulus value and spiking probability, or, in other words, the nonlinear tuning curve $P($ spike $\mid$ stim $=k)$. This single-feature curve quantifies the sensitivity of the neuron to the filtered stimulus independently of all other features. It is also possible to estimate an overall tuning relationship predicting the joint sensitivity of the neuron to all relevant stimulus features; however, this places significant demands on data sampling, and, for simplicity and statistical power, we worked only with separate curves corresponding to each individual feature. To estimate each curve, we applied Bayes' rule and related the probability of spiking conditioned on the stimulus value, $P($ spike $\mid$ stim $=k)$, to the probability of observing the stimulus value conditioned on the occurrence of a spike, $P($ stim $=k \mid$ spike $)$. According to Bayes' theorem, this relationship can be written as $P$ (spike $\mid$ stim $=$ $k) / P($ spike $)=P($ stim $=k \mid$ spike $) / P($ stim $=k)$. Given this equation, the tuning curve can be computed by taking the ratio of histograms of the spike-triggered stimulus distribution $P($ stim $=k \mid$ spike $)$ and the prior stimulus distribution $P(\operatorname{stim}=k$ ) (de Ruyter van Steveninck and Bialek, 1988; Brenner et al., 2000). We performed tuning curve estimates as described by Maravall et al. (2007). The analysis was repeated over 30 bootstrap samples of the filtered stimuli to produce the error bars shown in the tuning curve plots (error bars are the SD across bootstrap samples).

Presentation of results and correspondence across high-and low-variance epochs. STA or STC analyses were applied according to the following criteria (for an explanation of symmetric and rectified stimuli, see Results). For responses to symmetric stimuli, both STA and STC analyses were performed. For responses to rectified stimuli, STA analysis was conducted using the rectified stimulus waveform, whereas STC analysis was conducted using the original (nonrectified) stimulus waveform. This scheme for analyzing rectified stimuli was needed because of the following: (1) STC could not be usefully performed on rectified stimulus waveforms, because those waveforms were not Gaussian distributed, which could lead to artifacts (Brenner et al., 2000; Aguera y Arcas et al., 2003; Paninski, 2003; Bialek and De Ruyter Van Steveninck, 2005; Schwartz et al., 2006); and (2) STA analysis did not produce useful results on nonrectified stimulus waveforms because responses occurred to both positive and negative stimulus values, canceling out the STA.

The end result of our analysis was a set of two to three linear filters with their corresponding tuning curves for each neuron and condition. Comparisons across conditions (high vs low variance) were done as follows. High- and low-variance STA waveforms and their tuning curves were directly compared with each other. STC filters were first inspected visually to verify how they corresponded across conditions [the STC procedure implies that the first and second filters might be flipped across conditions (Aguera y Arcas et al., 2003; Bialek and De Ruyter Van Steveninck, 2005)]. For presentation of results, we surveyed the dataset to verify which feature usually contributed most significantly to the response of a neuron. Assessed by checking the sharpness and dynamic range of tuning curves, the most significant feature was usually one of the STC filters. For presentation, we therefore used tuning curves corresponding to the best STC filter; however, our results on rescaling held 
across all tuning curves, whether computed for the STA or for the STC filters.

Gain rescaling factors. Neuronal filters and nonlinear tuning curves are unique up to an arbitrary scale factor, because a change in filter amplitude is reflected as a scaling change in the input to the tuning curve (i.e., in the $x$-axis of the curve) (Brenner et al., 2000; Chichilnisky, 2001; Kim and Rieke, 2001). We chose to normalize all neuronal filters to unit norm, so that any change in the overall scale of the input-output relationship appeared as a change in the scale of the $x$-axis of the tuning curve.

To assess the amount of gain rescaling in tuning curves with respect to a change in stimulus SD, we fit seventh- or ninth-degree polynomials separately to the high- and low-variance tuning curves of each neuron. We then rescaled the $x$-axis of one fitted tuning curve by a factor $f$, solving for the value of $f$ that gave maximum overlap with the other curve. The resulting $f$ value was normalized such that an amount of $100 \%$ corresponded to the ratio of high and low SDs. In other words, a factor of $100 \%$ means that the curves had full rescaling, compensating for the change in stimulus statistics. Conversely, a value of $0 \%$ indicates that there was no rescaling. We also report the error value achieved by the best overlap, computed as the sum of squared differences between the normalized $y$-values of the curves for optimal $f$. Hence, perfect full gain rescaling would give a factor equal to $100 \%$ with an error equal to zero, precise rescaling could correspond to a factor close to $100 \%$ but with larger error, and a complete lack of rescaling would give a factor equal to $0 \%$ with a large error value.

Afterhyperpolarizations. Afterhyperpolarization magnitude was assessed by measuring membrane potential values with respect to baseline, at various time points after the end of pulse train stimuli (described above, Stimulus design). To arrive at a robust, standardized measurement of slow AHP amplitude across experiments, we chose the time point $400 \mathrm{~ms}$ after the end of the pulse train (Maravall et al., 2004). AHPs measured shortly after the end of a stimulus (e.g., $<100 \mathrm{~ms}$ after stimulus, or at the overall AHP peak) may include contributions from faster currents such as medium-duration AHPs, unrelated to the slower current of interest. We thus expected that measurements at a later time point such as $400 \mathrm{~ms}$, when the sAHP is the principal contributor to the overall AHP, would provide a more specific estimate of sAHP magnitude. Also, AHP amplitudes at $400 \mathrm{~ms}$ after stimulus proved less noisy across trials than the integrated area of the AHP. Standardized pulse train protocols provide a convenient way to generate AHPs that can be compared across neurons; to verify that sAHPs generated by standardized pulse trains were a good measure of those generated by white noise stimuli, we also measured voltage deflections $400 \mathrm{~ms}$ after the end of white noise stimulation.

Spike threshold estimation. Sodium current availability at different moments during the variance switching cycle was determined by measuring spike thresholds. We computed these by estimating the distribution of local membrane potential maxima (Kim and Rieke, 2003). This distribution falls off sharply at the spike threshold and has a small secondary "bump" at the peak spike membrane potential, because membrane potential values greater (more depolarized) than the spike threshold will not be local maxima unless they correspond to the spike peak. A good estimate of the spike threshold is therefore the membrane potential value at which the distribution falls off, which we extracted by taking the 99th percentile value of the distribution, a choice that gave robust results across neurons and conditions. The calculation was done separately for high- and low-variance periods. To check our results, we repeated the calculation using a different method: we estimated the threshold of each spike as the value of the membrane potential at the point of maximum acceleration of the action potential waveform and then averaged over all spikes. Again, this was done separately for high- and low-variance periods. The two methods gave fully consistent results; for figure presentation, we used data from the local maxima distribution method.

Effects of whole-cell access. Variations in whole-cell access can affect readings of action potential threshold and of voltage responses. For example, for rectified stimuli, the mean current injected during highvariance periods was greater than the mean current during low-variance periods. Hence, observed differences in high- and low-variance thresh- olds could be contaminated by the electrode depolarization, resulting in a greater apparent difference in threshold for recordings with poorer access. We verified that this was not the case by checking that there was no correlation between access resistance and threshold difference $(n=$ 34; $p=0.52$; Pearson's $\left.r^{2}=0.013\right)$. Similarly, there was no correlation between access resistance and measured sAHP magnitude ( $n=34$; $p=$ 0.18; Pearson's $r^{2}=0.055$ ).

Statistical data presentation. Statistical tests used are identified below together with their corresponding results; use of parametric tests always depended on whether distributions were not found to depart significantly from normality, as judged by the Lilliefors test. Significance criteria were set at $p=0.05$. Unless otherwise specified, results are quoted as mean \pm SEM.

\section{Results \\ Reproducing features of in vivo adaptation to stimulus statistics in slices}

To determine whether adaptation to complex stimuli and gain rescaling of input-output functions can arise from the intrinsic properties of barrel cortex neurons, we performed experiments in acute slices, exploiting some of the advantages of the preparation, such as ease of drug delivery. We measured adaptation in pyramidal neurons with whole-cell current-clamp recordings while injecting stochastic current waveform stimuli through the recording electrode. Each stimulus consisted of continuous, filtered white noise, generated by sampling from a Gaussian distribution of current values (for a full description, see Materials and Methods). To assess how responses to a stimulus depended on stimulation history and on the context within which the stimulus was presented, we used an experimental design in which stimulus variations occurred over two timescales (Smirnakis et al., 1997). The faster timescale involved variations in the instantaneous current value, which fluctuated with a correlation time $\sim 2 \mathrm{~ms}$, as determined by the width of the low-pass filter applied to the white noise stimuli. The slower timescale corresponded to changes in the overall distribution of current values: the variance of the distribution switched back and forth between a higher and a lower value at intervals of $2.5 \mathrm{~s}$ (i.e., the total duration of the varianceswitching cycle was $5 \mathrm{~s}$ ). An example stimulus waveform switching across high and low variances is shown in Figure $1 \mathrm{~A}$.

Barrel cortex neurons in the intact animal are strongly responsive to changes in the statistics of sensory stimuli (Maravall et al., 2007). During the course of our variance switching cycle, we expected neurons to be generally strongly excited immediately after switches from low to high variance and to reduce their response after switches from high to low variance. Adaptation would be defined by a decrease or accommodation in firing rate during the course of high-variance periods and an increase or recovery in rate during low-variance periods.

We first recorded responses while applying stimuli with a mean of $0 \mathrm{pA}$, with no additional direct current (DC) holding current (Fig. 1A). Because the stimuli were sampled from a Gaussian distribution, they were symmetrically distributed about their mean, with positive and negative current values being equally probable. We thus refer to these stimuli as "symmetric." Stimulus SDs were chosen so that neurons would fire at rates comparable with previous recordings of in vivo responses to white noise whisker stimulation $(\sim 1-5 \mathrm{~Hz}$; at least $1 \mathrm{~Hz}$ on average during low variance) (Maravall et al., 2007). According to this rule, SD during high-variance periods was set to between 100 and 300 pA depending on the neuron; across the population included in Figure 1, the mean SD was $175 \mathrm{pA}$. For these recordings and for most of the recordings in the study, SD for low-variance periods was equal to 0.7 times that for high-variance periods. 

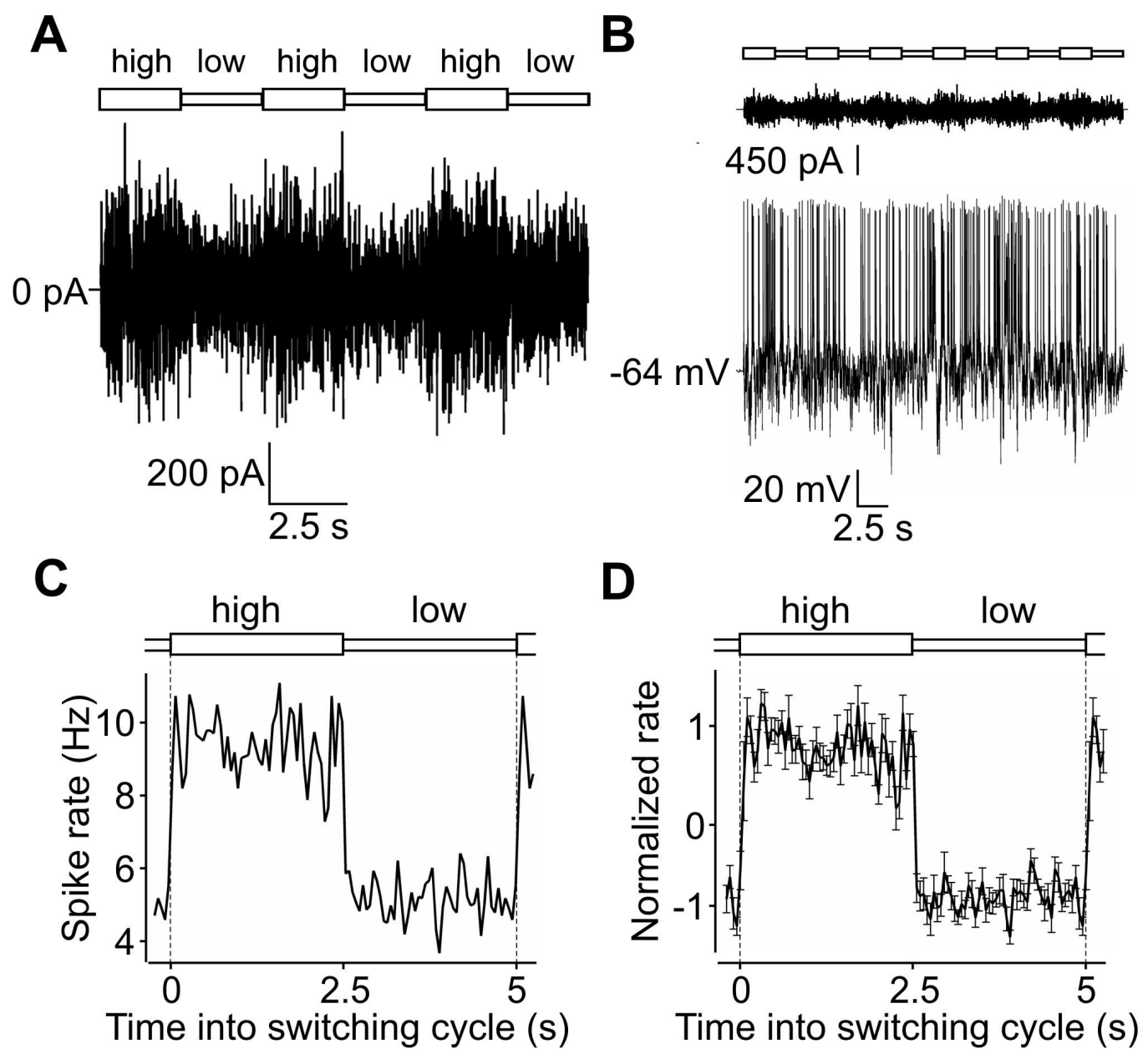

Figure 1. Absence of adaptation to stimulus statistics under symmetric current stimuli in barrel cortex. $\boldsymbol{A}$, Stimulus segment showing three $5 \mathrm{~s}$ cycles of high/low variance switching. High- and low-variance epochs are represented in the top diagram. Stimulus was distributed as a Gaussian and was symmetric about its $0 \mathrm{pA}$ mean. $\boldsymbol{B}$, High- and low-variance epochs (diagram as for $\boldsymbol{A}$; top trace), stimulus (current; middle trace), and response (membrane potential; bottom trace) for a typical neuron. Traces depict a complete trial. Numerical value at left indicates resting membrane potential. The neuron fired strongly during high-variance epochs and more weakly during low-variance epochs. $\boldsymbol{C}$, Firing rate plot for same neuron as $\boldsymbol{B}$, showing an absence of rate adaptation. The plot was constructed by binning spike times within each cycle (using 50 ms windows) and averaging responses over the number of cycles in the experiment. High- and low-variance epochs are represented in the top diagram; the transition from low to high is shown at both edges to emphasize the cyclic character of the plot. $\boldsymbol{D}$, Population pooled firing rate plot, showing normalized firing rate averaged over neurons ( $n=16$ ). Error bars represent 1 SD. Adaptation was absent across the population. $E$, Adaptation ratio plot (see Materials and Methods). Dark symbol depicts neuron in $\boldsymbol{B}$ and $\boldsymbol{C}$. Adaptation ratios $>1$ signified adaptation; in this population, ratios were somewhat, but not significantly, $<1$.

Under these conditions, we found that changes in variance caused neurons to respond robustly and stably throughout recordings lasting up to $2-3 \mathrm{~h}$ (Fig. $1 \mathrm{~B}$ ). The firing rates of neurons were thus strongly modulated by changes in variance. However, neurons typically showed no rate adaptation (Fig. $1 C, D$ ); the rate remained essentially the same within each high- or low-variance epoch. To measure rate adaptation and compare its magnitude across neurons, we defined an adaptation ratio, computed from the firing rates immediately after variance switches and at steady state; adaptation would correspond to a ratio value significantly $>1$ (see Materials and Methods). Across the dataset, adaptation ratios were smaller than 1 , although not significantly different $(0.94 \pm 0.03 ; n=16 ; p=0.056, t$ test) (Fig. $1 E)$.

This lack of adaptation contrasted with the prominence of the phenomenon in vivo. One plausible explanation for the absence of adaptation could have been that it has a purely synaptic basis that did not contribute to the responses recorded in slices. However, we still found our results puzzling, because previous work had found some amount of adaptation in cortical neurons under conditions apparently similar to our own, except for minor differences in stimulation protocol (Paninski et al., 2003). This sug- gested that perhaps subtle differences in stimuli can affect how neurons adapt to changes in stimulus context. We noted that several salient features of in vivo stimulation might affect adaptation. First, when stimulated with whisker motion in any direction, barrel cortex neurons in vivo receive net depolarizing synaptic inputs (Wilent and Contreras, 2005). Second, cortical responses to whisker white noise stimuli in vivo can show bilateral symmetry (Arabzadeh et al., 2005; Hasenstaub et al., 2007; Maravall et al., 2007). Hence, we reasoned that complex patterns of sensory stimulation in vivo may be more similar to rectified (net depolarizing) current waveforms than to zero-mean, symmetric current waveforms. We thus next tested adaptation to rectified stimulus waveforms, interleaving symmetric and rectified stimuli in a different set of experiments $(n=16)$. Rectified stimuli were generated by taking the absolute value of the symmetric white noise stimuli presented above.

Rectified waveforms caused clear adaptation (Fig. 2). Neurons typically showed significant adaptive modulation of firing rate during both high- and low-variance periods (Fig. $2 B, C$ ), and their adaptation ratios were significantly $>1(1.71 \pm 0.12 ; n=16$; $p<0.001, t$ test) (Fig. 2D). Ratios were also significantly greater 
A

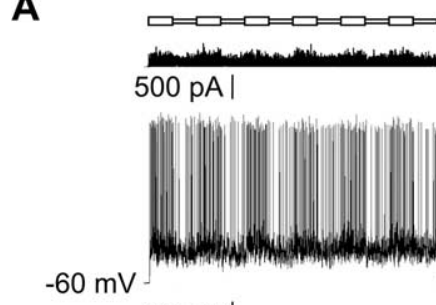

$20 \mathrm{mVL}$

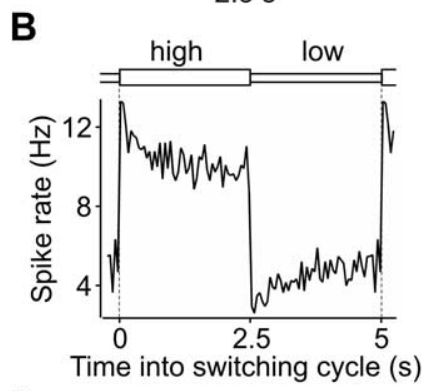

D

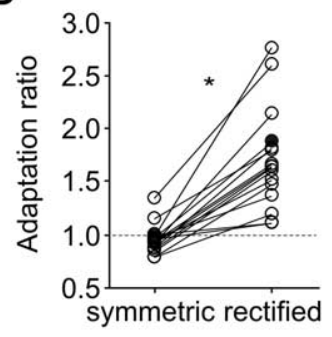

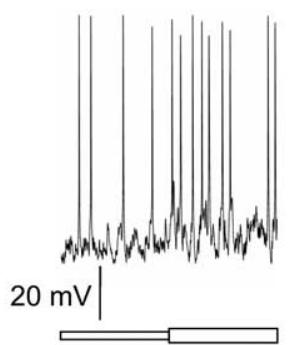

C

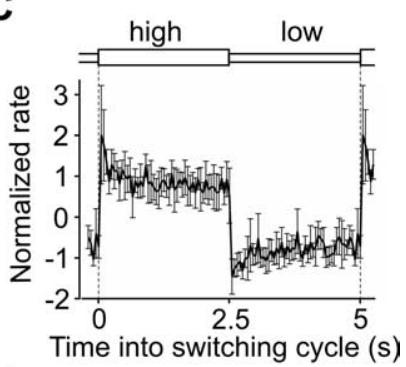

E

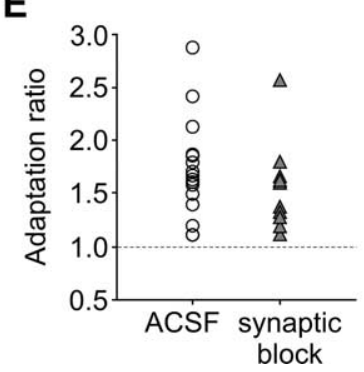

Figure 2. Adaptation to stimulus statistics under rectified stimuli. $\boldsymbol{A}$, Left, High- and lowvariance epochs (top), stimulus (current; middle), and response (membrane potential; bottom) for rectified stimuli, for a different neuron to that in Figure 1B. Right, Magnified membrane potential response, -80 to $+80 \mathrm{~ms}$ relative to a low- to high-variance transition. Bottom diagram indicates low-and high-variance periods. Length of bars, $80 \mathrm{~ms}$. Note tendency toward higher firing threshold during high-variance portion of trace. $\boldsymbol{B}$, Firing rate plot for same neuron as $\boldsymbol{A}$, constructed as Figure $1 C$, showing clear adaptation to rectified stimuli. $C$, Population rate plot $(n=16)$ showing significant adaptation to rectified stimuli. Error bars represent 1SD. $\boldsymbol{D}$, Adaptation ratio plot comparing ratio values across symmetric and rectified conditions. Lines connect symmetric and rectified data points for each neuron $(n=16)$. Asterisk denotes significant difference; also, ratios for rectified stimuli were significantly $>1$. Dark symbol depicts neuron in $\boldsymbol{A}$ and $\boldsymbol{B}$. $\boldsymbol{E}$, Adaptation was unchanged in the presence of synaptic blockers. Symbols and colors arbitrarily chosen to aid discrimination of data points.

for rectified than for nonrectified, symmetric stimuli $(0.93 \pm 0.02$ for symmetric; $n=16 ; p<0.001$, paired $t$ test) (Fig. $2 D$ ). The magnitude of adaptation was less than measured in vivo, which was unsurprising because contributions from synaptic depression and its recovery, which are likely to be present in the intact animal, were bypassed in our slice recordings. This caused steadystate firing rates in high-variance epochs to be greater than in vivo, even for neurons whose low-variance firing rates were comparable with typical levels in vivo. Strikingly, however, the timescale of rate adaptation with rectified stimuli was $330 \pm 40 \mathrm{~ms}$ $(n=16)$, close to that observed in vivo [280 $\pm 180 \mathrm{~ms}$ (Maravall et al., 2007)] and longer than that for other forms of adaptation, such as adaptation to stimulus mean (see below). Therefore, responses to rectified current stimuli reproduced some essential features of firing rate adaptation in vivo.

To verify that this adaptation was attributable to intrinsic properties, we performed one set of experiments with synaptic transmission blocked via joint application of the NMDA antagonist $R S-\mathrm{CPP}(10 \mu \mathrm{M})$ and the AMPA/kainate antagonist CNQX

$(10 \mu \mathrm{M})$. We observed no significant difference in adaptation relative to the control experiments $(1.56 \pm 0.13 ; n=10$ synaptic block experiments; $n=16$ control experiments; $p=0.40, t$ test) (Fig. 2E). Therefore, the adaptation to stimulus statistics observed using rectified stimuli was attributable to intrinsic membrane properties.

\section{Adaptive gain rescaling with rectified stimuli}

To assess the effect of adaptation on the stimulus-response relationships of neurons, we characterized these by extracting neuronal filters with spike-triggered average and covariance analysis and constructing input-output tuning functions separately for high- and low-variance periods (Fig. 3A) (see Materials and Methods). Both for symmetric stimuli and rectified stimuli, neuronal filters did not change across high- and low-variance periods (data not shown).

Using symmetric stimuli, input-output functions for highand low-variance periods were not identical (Fig. 3B). This implied that some amount of adaptive change in input-output functions occurred across changes in stimulus distribution, although the change was usually quite small. Some amount of gain rescaling can be expected even for integrate-and-fire models (Rudd and Brown, 1997; Aguera y Arcas and Fairhall, 2003; Paninski et al., 2003). To better visualize the extent of changes in tuning curves, we normalized the plots so that response probability appeared in terms of mean spike rate and input value appeared in units of the SD of the current distribution (i.e., as a $z$-score). We never found that curves overlapped when plotted in this way (Fig. 3C). This implied that changes in input-output function were not enough to compensate for the changes in stimulus distribution; in other words, tuning curves did not rescale their gain to adjust to the stimulus range.

We next determined tuning curves for responses to rectified stimuli. Plotted in absolute units, the widths of the tuning curves now diverged more clearly than for symmetric stimuli (Fig. 3D). Indeed, repeating the normalization procedure described above revealed that tuning curves plotted in relative units now overlapped (Fig. 3E). This meant that the change in tuning curve caused by switches in rectified stimulus statistics was just enough to compensate for the change in stimulus distribution. This matched the adaptive behavior observed under sensory stimulation in vivo (Maravall et al., 2007).

We extended this analysis of tuning curves to the population of neurons that were tested with both symmetric and rectified stimuli, examining whether rectification affected the amount of gain rescaling. For each neuron, we fit a polynomial to the tuning curves and rescaled it along the $x$-axis, looking for the rescaling factor that gave the most overlap (least error) between high- and low-variance curves. We set the rescaling factor to be $100 \%$ for perfect rescaling and $0 \%$ for no rescaling (see Materials and Methods). The optimal factor for tuning curves to symmetric stimuli diverged widely across neurons (Fig. $3 F$ ) and differed significantly from $100 \%$ (36 $\pm 9 \% ; n=16 ; p<0.001$, sign test). The best rescaling factor still often produced a considerable error (Fig. $3 G$ ). Neurons did, however, show partial gain rescaling with symmetric stimuli, because the optimal factor was usually $>0 \%$. Conversely, for rectified stimuli, the optimal factor was always close to $100 \%$ ( $93 \pm 3 \% ; n=16$; not significantly different from $100 \%, p=0.12$, sign test) and was significantly different from that for symmetric stimuli $(p<0.001$, Kolmogorov-Smirnov test) (Fig. $3 F)$. Error values for rectified stimuli were significantly better constrained ( $p<0.001$, Kolmogorov-Smirnov) (Fig. 3G). 
A
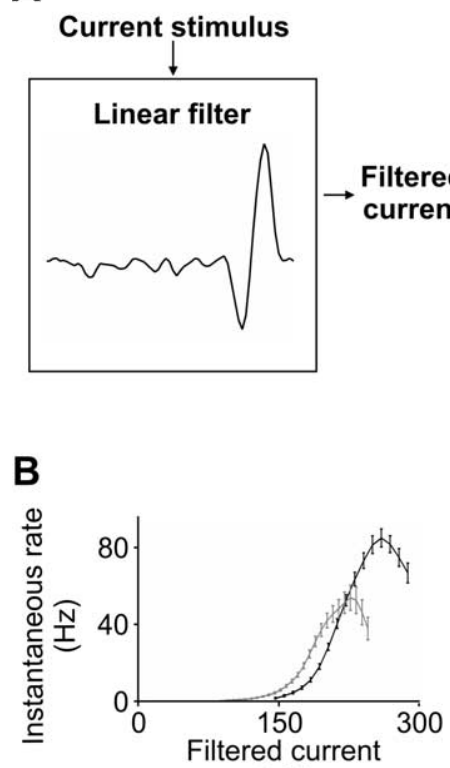

(pA)

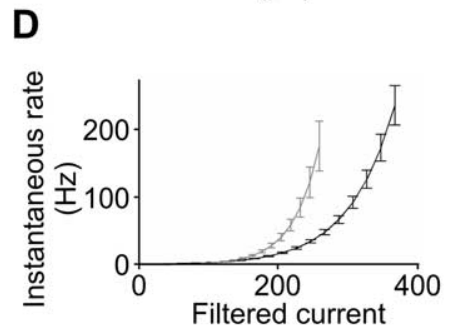

(pA)

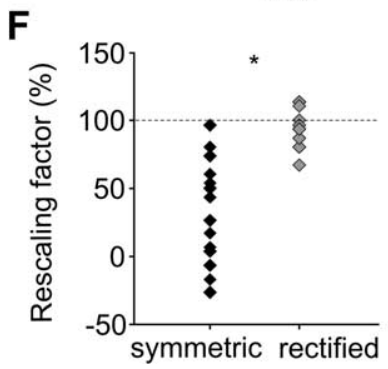

\section{C}

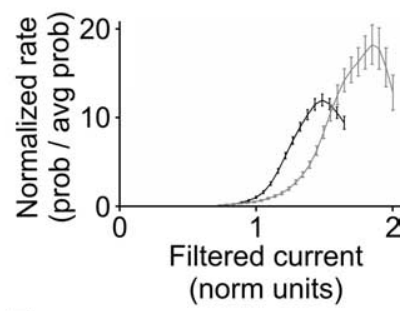

E

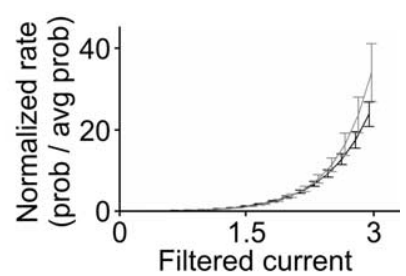

$\mathbf{G}$

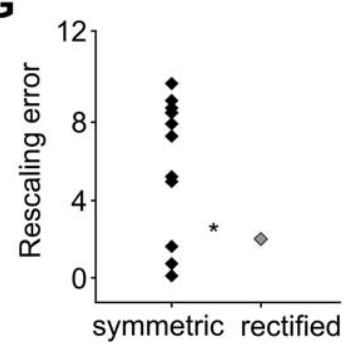

Figure 3. Full gain rescaling occurred only for rectified stimuli. A, Schematic of LN framework for characterizing neuronal stimulus-response relationships. Examples of linear filter and tuning curve correspond to the same neuron as the panels below, tested with rectified stimuli. $B$, Absolute input-output functions, or tuning curves, for neuron in Figure $2 A$ tested with symmetric stimuli. Curves were computed separately for high-variance epoch (dark line) and low-variance epoch (light line). Output is the predicted instantaneous firing probability in units of rate; input is the current stimulus projected onto the most significant neuronal filter from spike-triggered analysis (see Materials and Methods). Error bars represent SD from 30 repetitions of the estimation procedure. Note slight rescaling along $x$-axis: over values up to $\sim 200$ $\mathrm{pA}$, more current was necessary to reach a given firing rate in the high-variance epoch. The curve folds over for higher values. $\boldsymbol{C}$, Normalized input- output functions for same recording as in $\boldsymbol{B}$. Output is normalized to the average rate, thus representing how the input modulates the spiking probability of the neuron relative to its average; input is normalized by the SD of the current distribution. Rescaling is clearly not complete. $\boldsymbol{D}$, Absolute input- output functions for same neuron as in $\boldsymbol{B}$ and $\boldsymbol{C}$ tested with rectified stimuli. The width of the curves depended more strongly on the width of the distribution; in the high-variance epoch, significantly more current was required to achieve a given rate. $\boldsymbol{E}$, Normalized input- output functions, computed as in $\boldsymbol{C}$, show complete rescaling. $\boldsymbol{F}$, Rescaling factors across the same neuronal population as in Figure 2, tested with symmetric and rectified stimuli. For each neuron, the high-variance fitted tuning curve was multiplicatively rescaled along the $x$-axis until it best resembled the low-variance curve; plot depicts the resulting best rescaling factor, in units such that $100 \%$ (dashed line) corresponds to full rescaling and $0 \%$ to no rescaling. Factors under rectified stimulation, but not symmetric stimulation, clustered closely around $100 \%$. Here and in $\mathbf{G}$, asterisk denotes

This implies that, on average, neurons produced essentially full gain rescaling for rectified stimuli but not for symmetric stimuli.

\section{Preservation of adaptive gain rescaling across different SD ratios}

The above results show that, for rectified stimuli with a ratio of SDs equal to 0.7 , neurons produced adaptive gain rescaling that was just enough to compensate for the change in SD. We next asked whether this property still held for a different ratio of SDs.

We tested a set of neurons with symmetric and rectified stimuli whose lower SD was 0.5 times their higher SD instead of 0.7 times (Fig. 4). On average, these stimuli produced remarkably similar behavior: average normalized rate plots for 0.5 and for 0.7 almost overlapped with each other (Fig. $4 A-D$ ). As in the 0.7 case, symmetric stimuli with the 0.5 ratio did not cause adaptation $(0.90 \pm 0.02 ; n=8$; not significantly different from $1, p=$ $0.29)$ (Fig. $4 B, E$ ). Rectified stimuli again caused greater adaptation than symmetric stimuli $(3.01 \pm 0.52 ; n=8 ; p=0.0044$, paired $t$ test) (Fig. $4 D, E$ ). Not surprisingly, stimuli with the 0.5 ratio, which implied a larger step in SD, tended to produce greater adaptation than stimuli with the 0.7 ratio (values as above; $p=0.0025, t$ test). The timescale for 0.5 rate adaptation was longer than that for 0.7 rate adaptation, although not significantly so [timescale for 0.7 equal to $330 \pm 40 \mathrm{~ms}$, as above $(n=$ 16); timescale for 0.5 equal to $460 \pm 90 \mathrm{~ms}(n=8) ; p=0.13, t$ test].

Finally, we extended the "optimal rescaling factor" analysis presented above to rectified stimuli with the 0.5 ratio. With symmetric stimuli, the best rescaling factor again varied widely $(64 \pm$ $32 \% ; n=8$ ) (Fig. $4 F$ ) and tended to produce a large error (Fig. $4 G)$. Neither factors nor errors were significantly different from those for symmetric stimuli with the 0.7 ratio ( $p=0.98$ for both, Kolmogorov-Smirnov test). Of greater interest, responses to rectified stimuli with the 0.5 ratio still showed full rescaling, just as for the 0.7 ratio. The optimal rescaling factor was $93 \pm 2 \%(n=$ 8 ), greater than that for symmetric stimuli ( $p=0.01$, Kolmogorov-Smirnov test) (Fig. $4 F$ ) but identical to that for the 0.7 ratio (93 $\pm 3 \%$, as above; $n=16 ; p=0.84$, Kolmogorov-Smirnov test). Error values were again significantly smaller than for symmetric stimuli ( $p=0.01$, Kolmogorov-Smirnov test) (Fig. $4 G$ ). Therefore, responses to rectified stimuli had full adaptive gain rescaling, compensating for changes in stimulus SD across a range of at least a factor of 2 .

\section{Stimulus specificity of adaptation}

Our rectified stimuli had the property that a switch in variance also caused a switch in mean. Correspondingly, barrel cortex neurons in vivo are sensitive to absolute whisker velocity, and changes in velocity variance may also change the mean of the stimulus effectively driving them: neurons are more strongly driven by higher-variance stimuli (Maravall et al., 2007). We therefore next asked whether the enhanced adaptation caused by rectified stimuli was specific to this particular form of stimulation, i.e., unique to stimuli incorporating joint switches in both mean and variance. The alternative possibility was that rate ad-

$\leftarrow$

significant difference across distributions. G, Error values for rescaling across the population. Error values were computed from the difference between tuning curves after applying the optimal rescaling factors shown in $\boldsymbol{F}$. Perfect rescaling would give a negligible error. Rectified stimulation, but not symmetric stimulation, gave well constrained errors. Not shown (out of scale) are four outlying values $(>30)$ corresponding to symmetric stimuli. 
A

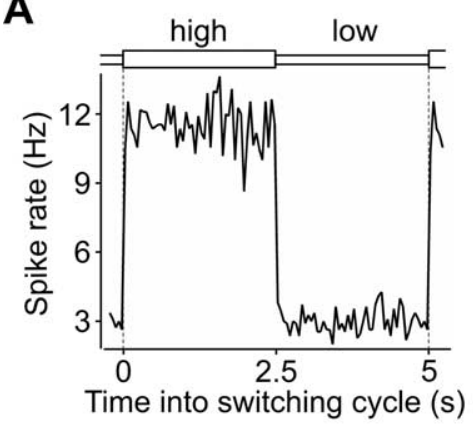

B

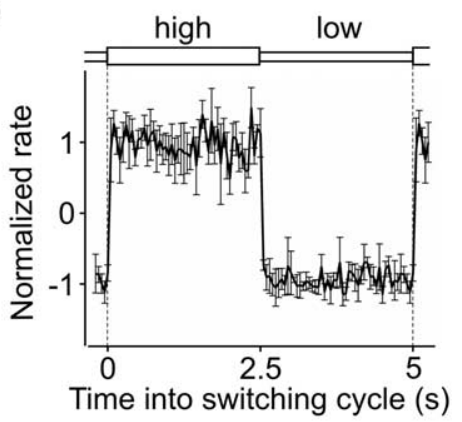

C

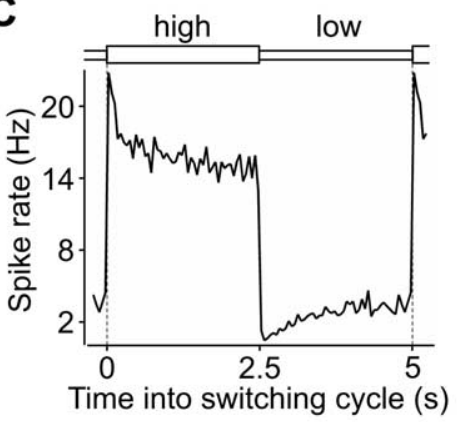

D

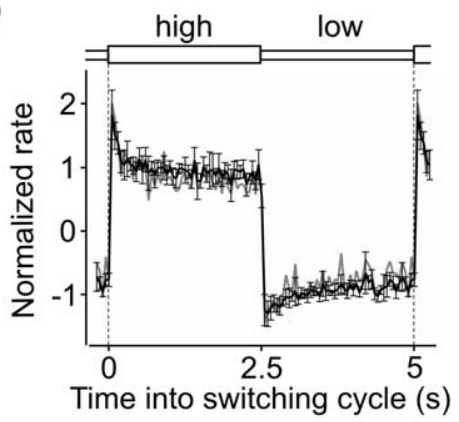

E

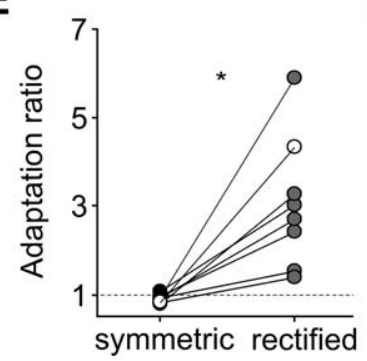

$\mathbf{F}$

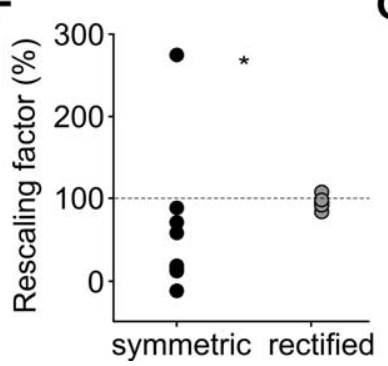

G

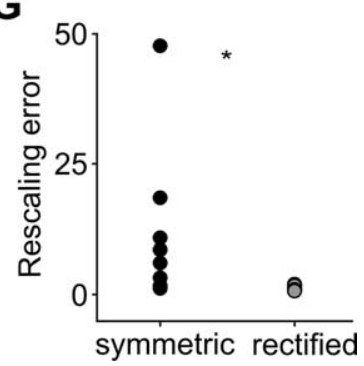

Figure 4. Preservation of adaptation and gain rescaling under rectified stimuli for a different ratio of SDs. A, Firing rate plot for a neuron, constructed as Figure 1, showing absence of adaptation to symmetric stimuli with a low- to high-SD ratio equal to 0.5 . $\boldsymbol{B}$, Population rate plot for symmetric stimuli $(n=8)$ showing absence of adaptation. Here and in $\boldsymbol{D}$, error bars represent 1 SD. $\boldsymbol{C}$, Rate plot for same neuron as $\boldsymbol{A}$, showing pronounced adaptation to rectified stimuli with a low- to high-SD ratio equal to 0.5 . $\boldsymbol{D}$, Population rate plot for rectified stimuli $(n=8)$ showing significant adaptation. Also shown (gray line) is the average population rate for neurons tested with a ratio of SDs equal to 0.7 . The gray and black lines overlap with each other, demonstrating the similarity of the behavior for different $S D$ ratio parameters. $E$, Adaptation ratio plot comparing ratio values across symmetric and rectified conditions. Lines connect symmetric and rectified data points for each neuron $(n=8)$. Asterisk denotes significant difference; also, ratios for rectified stimuli were significantly $>1$. White symbol depicts neuron in $\boldsymbol{A}$ and $\boldsymbol{C}$. $\boldsymbol{F}$, Rescaling factors across population, tested with symmetric and rectified stimuli. Asterisk denotes significant difference across distributions. Rescaling factors computed as in Figure 3F. G, Error values for rescaling across population. Rectified stimulation, but not symmetric stimulation, gave well constrained errors. Errors computed as in Figure 3G.

aptation with gain rescaling was not specific to rectified stimuli but was common to other forms of stimulation (i.e., was generic). This could happen if rectified stimuli evoked more adaptation than zero-mean stimuli simply because they drove neurons more strongly, e.g., by depolarizing them more or by evoking spikes at a frequency high enough to activate relevant mechanisms. This possibility would predict that the patterns of adaptation caused by rectified stimuli could be reproduced with other forms of stimulation, as long as neurons were comparably excited. To distinguish between the two possibilities, we applied stimuli that varied independently in mean and variance and added DCs as required. In these experiments, different stimulus types were always interleaved, and the ratio of SDs was always 0.7 as in the experiments of Figures 1-3.

First, we used current stimuli whose mean switched across high and low values, over a cycle duration equal to that for the variance-switching stimuli used previously. For each neuron, mean-switching stimuli had an SD equal to that of lowvariance epochs of the variance-switching rectified stimuli (setup as described above). The lower mean current value of the mean-switching stimulus was set to evoke the same membrane depolarization reached during low-variance epochs of the rectified stimuli. The magnitude of the step change in current across high-mean and low-mean stimuli was set to be equal to the step change in membrane potential induced by switches across high- and lowvariance rectified stimuli, divided by the input resistance of the neuron. We reasoned that this change should suffice to induce an instantaneous change in spiking rate comparable with that caused by switches in rectified stimuli, and that the resulting mean-switching stimulus would evoke peak firing rates and patterns of current activation similar to those generated by rectified stimuli. Strikingly, under these conditions, responses were strongly distinct from those evoked by rectified stimuli (Fig. 5, compare A1, A2). Across the population of neurons tested, changes in stimulus mean caused a sharp change in response, larger than that caused by rectified stimuli, followed by extremely strong and rapid rate adaptation (Fig. 5A3). Adaptation to switches in stimulus mean was therefore very different from adaptation to switching rectified stimuli, even when stimulus parameters were set to evoke comparable response magnitudes.

Next, we considered symmetric (nonrectified) current stimuli with switching variance, similar to the waveform depicted in Figure $1 \mathrm{~A}$, except that we now injected additional DC. The aim of this manipulation was to match the low-variance average membrane depolarization and firing rate to those reached with rectified stimuli. Because, as mentioned above, rectified stimuli implied a positive mean current, adaptation under rectified stimuli could have been attributable simply to the greater evoked depolarization and firing rate. Mean depolarizing current during rectified stimulation averaged $\sim 80 \mathrm{pA}$ across neurons (range, 30-200 pA): in added DC experiments, we provided a similar amount of depolarizing DC manually, supplementing the symmetric variance-switching white noise stimulus. The resulting responses had negligible adaptation (Fig. 5B); they were qualitatively more similar to responses to symmetric variance-switching stimuli (as in Fig. 1) than to responses to rectified stimuli (Fig. 5B1-B3) (see also Fig. 2). The difference with responses to rectified stimuli held across the population (adaptation ratio for rectified stimuli, $1.71 \pm 0.26$; for added DC stimuli, $1.04 \pm 0.04 ; n=7 ; p=0.0041$, Wilcoxon's rank sum test) (Fig. 5B4).

Therefore, adaptation depended strongly on the specific form of changes in the stimulus distribution. Forms of stimulation 

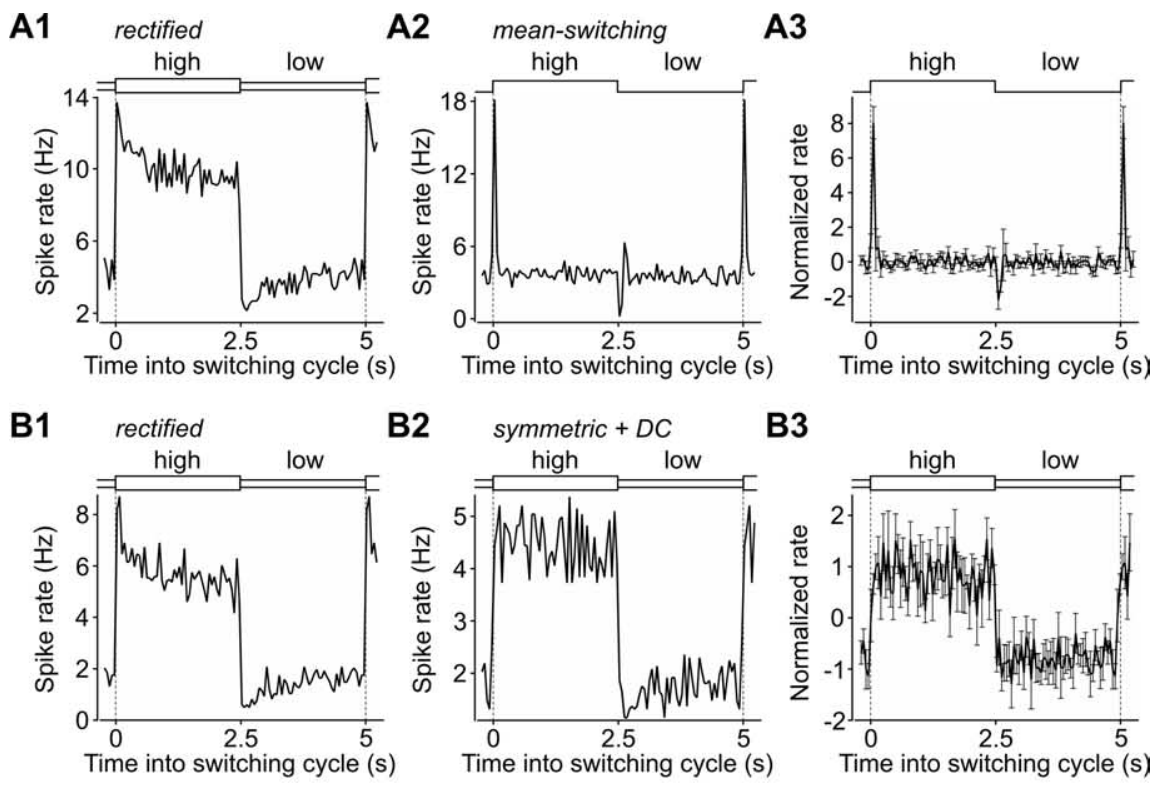

B4

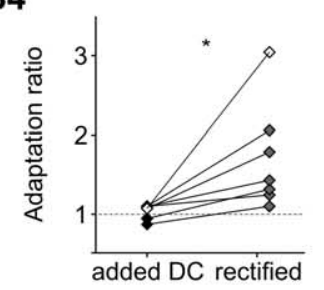

B5

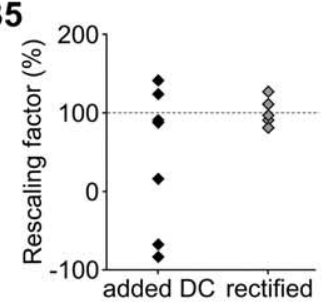

B6

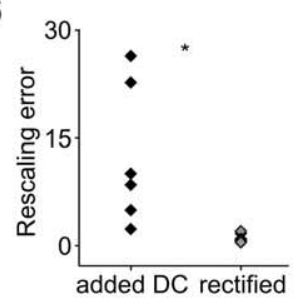

C1

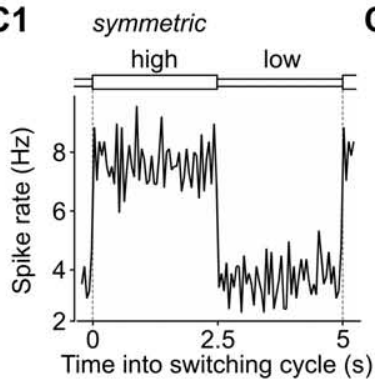

C4

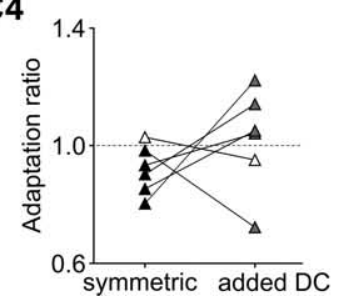

C2

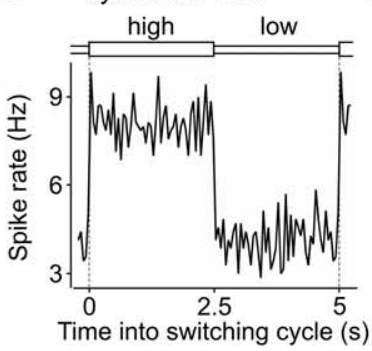

C5

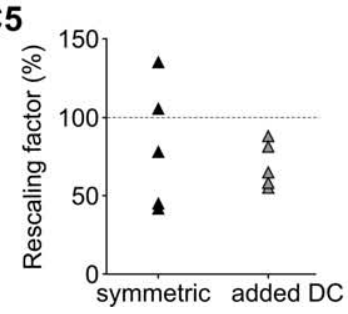

C3

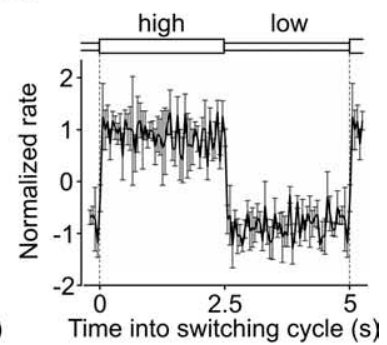

C6

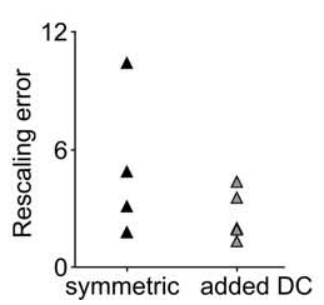

Figure 5. Stimulus dependence of adaptation. $A$, Comparison between responses to rectified stimuli and mean-switching stimuli. $\boldsymbol{A 1}, \boldsymbol{A 2}$, Rate plots for responses to rectified stimuli $(\boldsymbol{A} \mathbf{1})$ and mean-switching stimuli $(\boldsymbol{A 2})$ for one neuron. Plots constructed as in Figure $1 C$. Note the striking difference in rate adaptation characteristics, despite the similar firing rate reached at steady state during the "low" epoch. $\boldsymbol{A}$ 3, Population rate plot for mean-switching data $(n=8)$. Error bars depict 1 SD. $\boldsymbol{B}$, Comparison between responses to rectified stimuli and symmetric stimuli with added DC (for explanation, see Results). B1, B2, Rate plots for responses to rectified stimuli (B1) and added DC stimuli (B2) for a neuron different to the one in $\boldsymbol{A} \mathbf{1}$ and $\boldsymbol{A} \mathbf{2}$. Lack of adaptation under added $D C$ stimuli is similar to behavior under symmetric stimuli in Figure 1, despite the increased excitation provided by the $D C$. B3, Population rate plot for added DC data $(n=7)$. Error bars as in $\boldsymbol{A 3}$. B4, Adaptation ratios across population tested with rectified and added DC stimuli. Lines connect data points for each neuron $(n=7)$. Asterisk denotes significant difference. White symbol depicts neuron in $\boldsymbol{B} \mathbf{1}$ and $\boldsymbol{B} \mathbf{2}$. $\boldsymbol{B}$, Rescaling factors across population tested with rectified and added $D C$ stimuli. Factors computed as in Figure 3F. B6, Error values for rescaling across population tested with rectified and added DC stimuli. Asterisk denotes significant difference across distributions. Errors computed as in Figure 3G. C, Comparison between responses to symmetric stimuli without and with added DC (for explanation, see Results). C1, C2, Rate plots for responses to symmetric stimuli (C1) and added DC stimuli (C2) for a neuron different from those in $\mathbf{A 1}, \mathbf{A} \mathbf{2}$ and $\mathbf{B 1}, \mathbf{B 2}$. Lack of adaptation is similar with or without added DC. C3, Population rate plot for added DC data $(n=6)$. Error bars as in A3. C4, Adaptation ratios across population tested with symmetric and added DC stimuli. Lines connect data points for each neuron $(n=6)$. There was no causing matching firing rates or depolarization levels did not evoke similar forms of adaptation. In vivo-like rate adaptation only occurred when stimuli were rectified, implying joint switches in mean and variance.

We asked whether the amount of gain rescaling depended on the added DC using the same optimal rescaling factor method described above. For the set of neurons tested with rectified and added DC (Fig. $5 B$ ), there was full gain rescaling under rectified stimulation but only partial rescaling under added DC stimulation [rectified, $99 \pm 7 \%$; added DC, $44 \pm 34 \% ; n=7$; no significant difference in factor, $p=0.42$, Kolmogorov-Smirnov test (Fig. 5B5); but highly significant difference in error, $p<$ 0.001, Kolmogorov-Smirnov test (Fig. $5 B 6)]$.

We also tested an additional, separate set of neurons with a symmetric varianceswitching protocol with or without added DC (Fig. $5 C$ ). The principal aim of this dataset was to test whether adding DC would, in itself, enhance adaptation or gain rescaling compared with symmetric stimuli. We found that adding DC caused no significant or systematic changes (Fig. 5C1-C3). Adaptation was unaltered (symmetric, no DC, $0.92 \pm 0.03$; added DC, $1.02 \pm 0.07 ; n=6 ; p=0.34$, paired $t$ test) (Fig. 5C4). Gain rescaling was also unaltered [symmetric, no DC, $79 \pm 18 \%, n=5$; added DC, $66 \pm 6 \%, n=6$; no significant difference in factor, $p=0.65$, Kolmogorov-Smirnov test (Fig. 5C5); or in error, $p=$ 0.99 , Kolmogorov-Smirnov test (Fig. 5 C6)].

Taking these results together, adding DC did not affect the incomplete gain rescaling observed with symmetric stimuli. Rectifying stimuli did produce full rescaling. The characteristics of adaptation to rectified stimuli were only evoked by joint changes in mean and variance.

\section{Experimental analysis of adaptation to rectified stimuli}

Although most of our experiments were performed at room temperature, one set was performed at physiological temperature $\left(T=34 \pm 1^{\circ} \mathrm{C}\right)$. Adaptation was

$\leftarrow$

significant difference in adaptation. White symbol depicts neuron in C1 and C2. C5, Rescaling factors across population tested with symmetric and added DC stimuli. Factors computed as in Figure $3 F$. Adding DC did not significantly change the distribution of rescaling factors. C6, Error values for rescaling across population tested with symmetric and added DC stimuli. Errors computed as in Figure 3G. Adding DC made no difference to the distribution of rescaling errors. 
present in these experiments, i.e., the adaptation ratio was significantly $>1(1.51 \pm 0.09 ; n=9 ; p<0.001, t$ test) (Fig. 6A). Although ratios were somewhat smaller than for room temperature experiments, this modest reduction was not statistically significant (vs $n=16$ room temperature experiments; $p=0.26, t$ test). There was no significant change in the timescale of rate adaptation $(250 \pm 60 \mathrm{~ms} ; n=9 ; p=0.23$, $t$ test $)$.

We next asked whether the magnitude of rate adaptation varied across pyramidal neurons in different cortical layers. To examine this, we performed recordings with rectified stimuli and classified them into categories according to the layer location of the recorded cell (Fig. 6B). There was no difference between adaptation ratios for pyramidal neurons in layers $2 / 3$, layer 4 , and layer 5 (layers 2/3, $1.75 \pm 0.18, n=16$; layer $4,1.66 \pm 0.12, n=$ 10; layer 5, $1.77 \pm 0.12, n=18 ; p=0.74$, Kruskal-Wallis test). The behavior was therefore common to regular-spiking neurons located across different layers.

We further classified our dataset according to internal pipette solution. Different anions are known to have different effects on neuronal response properties as they dialyze the cell (Zhang et al., 1994; Velumian et al., 1997; Kaczorowski et al., 2007). Specifically, dialysis with potassium gluconate reduces sAHPs, whereas potassium methylsulfate and methylsulfonate maintain sAHP magnitude. The choice of anion affected adaptation: use of potassium gluconate solution (which also contained 1.1 mM EGTA) resulted in a decrease in rate adaptation compared with the other ions (gluconate, $1.50 \pm 0.07, n=14$; methylsulfate, $1.84 \pm 0.20$, $n=8$; methylsulfonate, $1.97 \pm 0.11, n=30 ; p=0.028$, ANOVA) (Fig. 6C).

\section{Strong correlation between adaptation and calcium-dependent sAHP}

Cortical neurons express calcium- and sodium-dependent potassium conductances that evoke sAHPs during spiking and induce spike-frequency adaptation under stimulation with DC pulses (Madison and Nicoll, 1984; Schwindt et al., 1988, 1989). As explained above, calcium-dependent mechanisms, particularly sAHPs, can be affected by the choice of anion used in whole-cell recordings, and we found a similar effect on adaptation. Reasoning that this suggested a likely link between sAHPs and adaptation, we analyzed this relationship using a standardized pulsetrain protocol to evoke and measure sAHPs (Fig. 7A) (see Materials and Methods). We first confirmed that sAHP magnitude (measured $400 \mathrm{~ms}$ after the end of stimulation) depended on the choice of internal anion, as in the existing literature (see above). Indeed, sAHPs recorded with methylsulfate or methylsulfonate in the pipette were larger than those with gluconate (gluconate, $-0.1 \pm 0.2 \mathrm{mV}, n=14$; methylsulfate, $-2.8 \pm 0.5$ $\mathrm{mV}, n=8$; methylsulfonate, $-3.1 \pm 0.2 \mathrm{mV}, n=30 ; p<0.001$, ANOVA) (Fig. 7A). We also checked the effects of temperature on the sAHP. As expected from the literature (Thompson et al., 1985; Lee et al., 2005), sAHP magnitude was smaller at physiological temperature $\left(T=34 \pm 1^{\circ} \mathrm{C}\right)$, although this effect did not reach statistical significance $\left(34^{\circ} \mathrm{C},-1.2 \pm 0.8 \mathrm{mV}, n=9\right.$; room temperature, $-2.1 \pm 0.4 \mathrm{mV}, n=16 ; p=0.26, t$ test $)$. The magnitude of adaptation was therefore linked with the magnitude of sAHPs in that they were both modulated by the choice of internal ion; both were also reduced at physiological temperature, although not significantly so.

To determine the extent to which calcium-dependent mechanisms contribute to adaptation, we blocked calcium entry via bath application of $\mathrm{CdCl}_{2}$ solution $(50 \mu \mathrm{M}) . \mathrm{CdCl}_{2}$ application abolished the sAHP in responses to pulse-train stimulation
$(-0.50 \pm 0.06 \mathrm{mV}, n=6$; vs ACSF, $-3.1 \pm 0.4 \mathrm{mV} ; p<0.001$, paired $t$ test) (Fig. $7 B$ ). Adaptation to rectified stimuli was reduced by $\mathrm{CdCl}_{2}$ (1.22 $\pm 0.07, n=6$; vs ACSF, $2.16 \pm 0.39 ; p=$ 0.040 , paired $t$ test) (Fig. 7C). However, adaptation ratios remained significantly higher than $1(n=6 ; p=0.026, t$ test $)$, implying that non-calcium-dependent mechanisms could play a role in adaptation.

Cadmium is a nonselective blocker of calcium-dependent properties. We also manipulated SAHPs via application of the serotonergic $\left(5-\mathrm{HT}_{2}\right)$ agonist $\alpha$ me5HT $(20 \mu \mathrm{M})$, which reduces sAHPs (Villalobos et al., 2005) and increases neuronal gain to DC stimulation (Zhang and Arsenault, 2005). We confirmed that sAHPs were significantly reduced by $\alpha$ me5HT $(-0.8 \pm 0.2 \mathrm{mV}$, $n=9$; vs ACSF, $-3.3 \pm 0.4 \mathrm{mV} ; p<0.001$, paired $t$ test) (Fig. $7 D)$. However, adaptation ratios under rectified stimulation were not significantly affected $(1.88 \pm 0.23, n=9$; vs ACSF, $1.96 \pm$ $0.14 ; p=0.76$, paired $t$ test) (Fig. $7 E$ ). Because serotonergic $5-\mathrm{HT}_{2}$ agonists act on many mechanisms, $\alpha$ me5HT may have affected other intrinsic properties that might also influence adaptation, compensating for its inhibition of the sAHP.

We further analyzed the relationship between sAHPs and adaptation by measuring correlations between sAHP magnitude and adaptation ratio in the main dataset (Fig. $7 F$ ). This provided an approach that was complementary to pharmacology and avoided possible problems of interpretation arising from nonselective effects of drugs. Methylsulfate-, methylsulfonate-, and gluconate-based recordings gave different results regarding both adaptation ratio and sAHP magnitude (see above); hence, we separated the three groups. Correlation analyses were performed on the gluconate group and the methylsulfonate group (which had the largest sizes) (Fig. 7F). We found that, for the methylsulfonate experiments, in which the sAHP was best maintained during whole-cell dialysis, sAHP magnitude and adaptation ratio under rectified stimulation were significantly correlated: SAHP magnitude accounted for $32 \%$ of the variance in the adaptation data $\left(n=30 ; p=0.0011\right.$; Pearson's $\left.r^{2}=0.32\right)$. Conversely, in gluconate experiments, both the sAHP and adaptation were depressed (see above) and what remained of adaptation depended on other mechanisms: there was no significant correlation between sAHP magnitude and adaptation $(n=14 ; p=0.39$; Pearson's $\left.r^{2}=0.062\right)$. The degree of adaptation to white noise stimulus statistics was therefore associated with the magnitude of sAHP currents, consistent with the pharmacology experiments described above.

We wondered whether the above assessment of the relationship between adaptation and sAHP magnitude was reliable. It was possible that our standardized pulse-train protocol for sAHP measurement did not adequately probe the slow currents participating in white noise adaptation. This issue might take two forms. First, the amount of calcium-dependent sAHP evoked by trains of brief pulses might not be a good predictor of the amount of calcium-dependent sAHP evoked with white noise stimulation, implying that the above measurements would produce an artifactually weak correlation between sAHP activation and adaptation to white noise. Consistent with this idea, previous work has found that cortical sAHP-related adaptation under fluctuating stimuli and irregular spike patterns is weaker than adaptation under regular stimulation with current pulses (Tang et al., 1997; Destexhe and Pare, 1999). Second, other slowly activating mechanisms beyond the calcium-dependent sAHP might come into play during long-lasting stimulation protocols (e.g., after several seconds of continuous current injection). For instance, in cat primary visual cortex, sAHPs caused by a sodium-dependent po- 
A

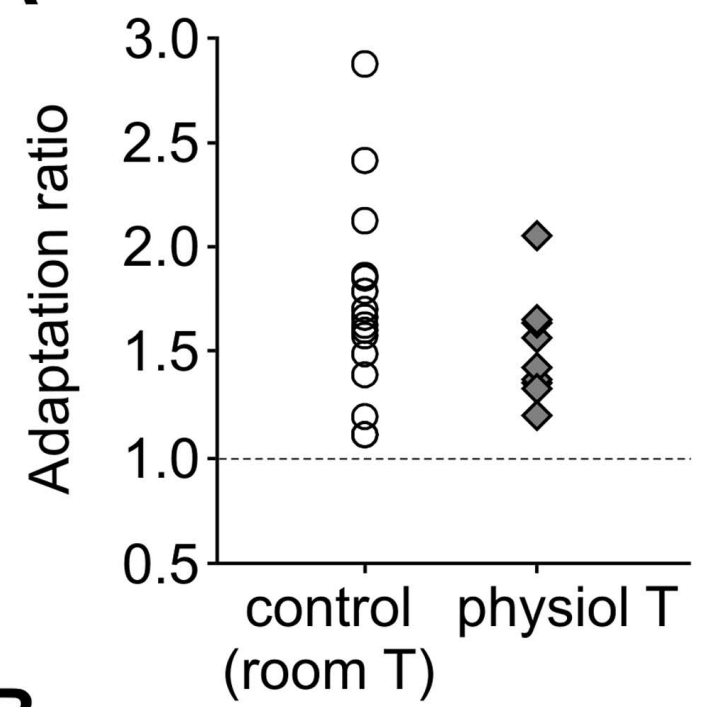

B

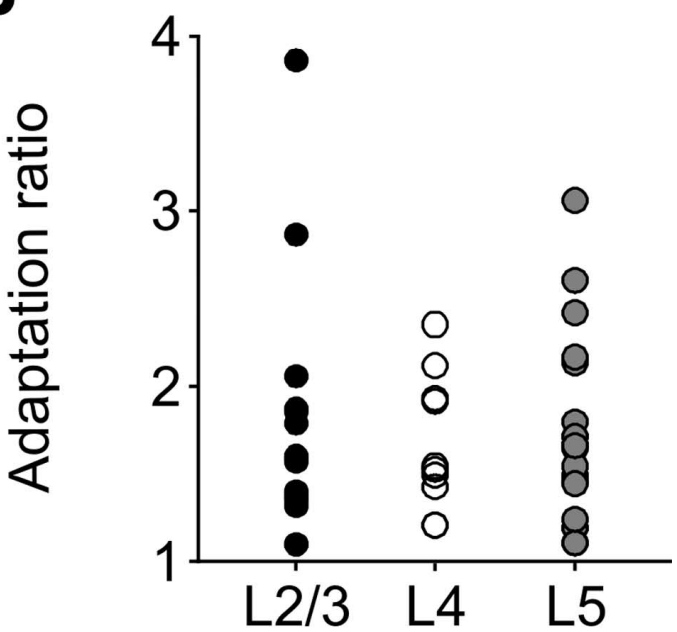

C

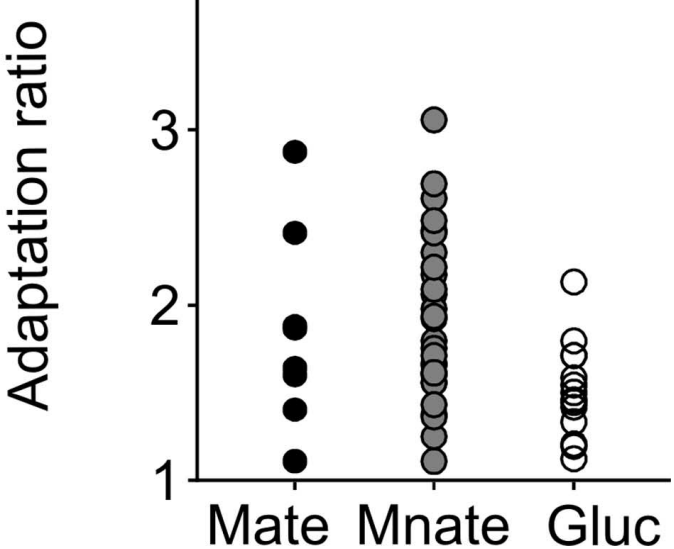

Figure 6. Experimental analysis of intrinsic adaptation. For all panels, symbols and colors were arbitrarily chosen to aid discrimination of data points. $\boldsymbol{A}$, Adaptation was present at physiological temperatures $\left(34 \pm 1^{\circ} \mathrm{C}\right)$, albeit slightly smaller in magnitude. $\boldsymbol{B}$, Adaptation was not significantly different across neurons from different cortical layers. $\boldsymbol{C}$, Adaptation was different across neurons recorded with different internal anions. Mate, Potassium methylsulfate recordings; Mnate, potassium methylsulfonate recordings; Gluc, potassium gluconate recordings. Asterisk denotes significant difference.
A

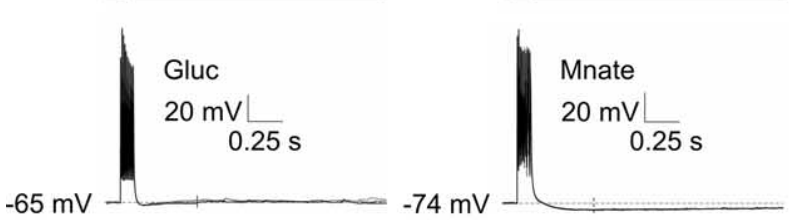

B

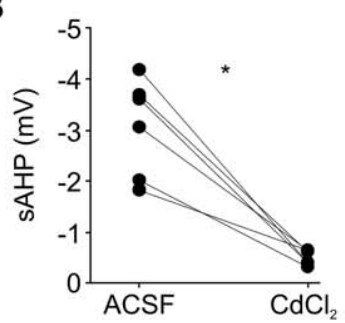

C
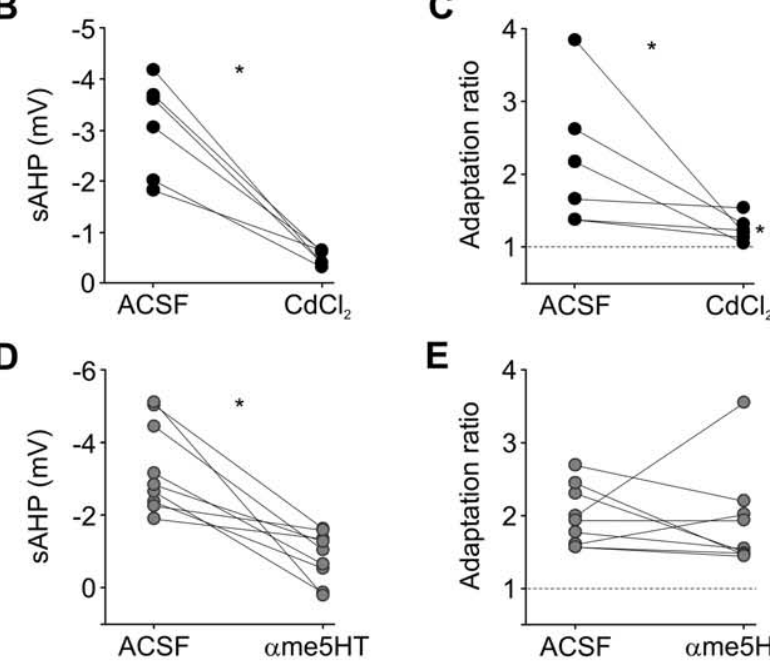

E

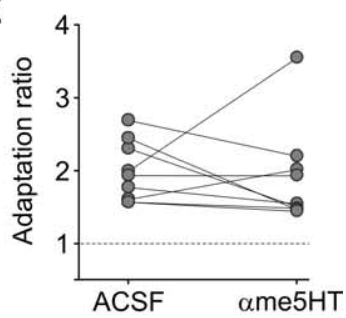

F

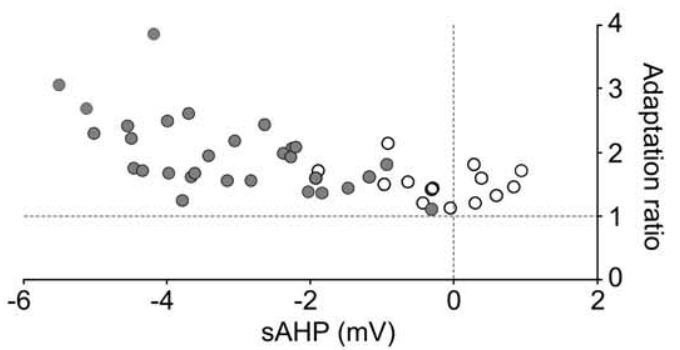

G

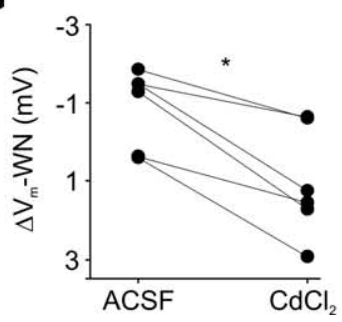

H

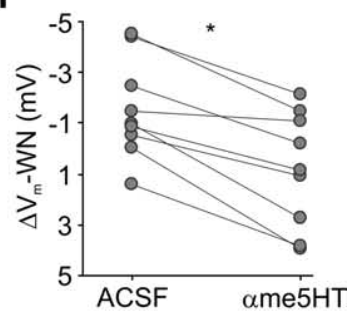

Figure 7. Strong, but not complete, correlation between adaptation and slow AHP current. For all panels, asterisks denote significant differences. $\boldsymbol{A}$, Standardized protocol for sAHP measurement: 10 current pulses delivered at $100 \mathrm{~Hz}$ (top traces). Voltage traces (bottom) are from a gluconate recording (Gluc; left) and a methylsulfonate recording (Mnate; right). Numerical values indicate resting membrane potential. Methylsulfonate recordings had larger sAHPs, which were measured $400 \mathrm{~ms}$ after the stimulus train (thin vertical lines show time point). $\boldsymbol{B}$, $\mathrm{CdCl}_{2}$ significantly decreased pulse train-evoked sAHPs. Lines connect the data points of each neuron $(n=6)$. $C, \mathrm{CdCl}_{2}$ significantly decreased adaptation under rectified stimulation, although adaptation ratios were still larger than 1 (right asterisk). $D, \alpha$ me5HT also significantly decreased pulse train-evoked sAHPs $(n=9)$. $\boldsymbol{E}$, However, $\alpha$ me5HT did not significantly reduce adaptation. $\boldsymbol{F}$, Adaptation ratios versus pulse train-evoked sAHPs. Gray symbols, Methylsulfonate recordings; white symbols, gluconate recordings. There was a significant correlation between adaptation ratio and SAHP for methylsulfonate, but not gluconate, recordings. Variations in the sAHP explained $32 \%$ of the variance in adaptation. $\mathbf{G}, \mathrm{CdCl}_{2}$ significantly decreased the average voltage deflection from baseline that remained $400 \mathrm{~ms}$ after the end of white noise stimuli, $\Delta V_{\mathrm{m}}-\mathrm{WN}(n=6) . \boldsymbol{H}, \alpha$ me5HT also significantly decreased $\Delta V_{\mathrm{m}}-\mathrm{WN}(n=9)$. 
tassium current underlie a form of slow contrast adaptation with a characteristic $\sim 10 \mathrm{~s}$ timescale (Sanchez-Vives et al., 2000b), although the mechanism has not been found previously to play a significant role in barrel cortex (Chung et al., 2002; Katz et al., 2006). We reasoned that, if either of these possibilities applied, voltage deflections at the end of long-lasting white noise stimuli would predict adaptation ratio values better than sAHPs evoked by standardized DC pulse trains. We therefore measured the average voltage deflection from baseline remaining $400 \mathrm{~ms}$ after the end of white noise trials for each neuron. Across the population, this quantity, termed $\Delta V_{\mathrm{m}}-\mathrm{WN}$, correlated with the sAHP measured with pulse sequences $\left(n=46 ; p<0.001\right.$, Pearson's $r^{2}=$ 0.26 ; data not shown), suggesting that it reflected similar mechanisms. Furthermore, $\Delta V_{\mathrm{m}}$-WN also decreased after application of $\mathrm{CdCl}_{2}(1.0 \pm 0.6 \mathrm{mV}, n=6$ vs ACSF, $-0.9 \pm 0.4 \mathrm{mV} ; p=$ 0.026 , Wilcoxon's test) (Fig. $7 G)$ and of $\alpha$ me5HT $(0.8 \pm 0.8 \mathrm{mV}$, $n=9$ vs ACSF, $-1.5 \pm 0.7 \mathrm{mV} ; p=0.03$, paired $t$ test) (Fig. $7 H$ ). (However, $\Delta V_{\mathrm{m}}-\mathrm{WN}$ values were sometimes positive rather than negative, suggesting contributions from other slowly activating mechanisms beyond the sAHP, such as afterdepolarizations.) Most importantly, $\Delta V_{\mathrm{m}}$-WN did not correlate with the adaptation ratio any better than did the sAHP evoked by pulse sequences (methylsulfonate recordings, $n=30 ; p=0.014$; Pearson's $r^{2}=$ 0.20 ; data not shown; adaptation ratio vs pulse-evoked sAHP shown in Fig. $7 F$ ). Thus, sAHPs evoked by pulse sequences provided a good measure of the overall voltage modulation caused by slowly activating currents. This implies that, out of those currents, calcium-dependent sAHP currents were the main contributor to adaptation to white noise stimulus statistics.

For independent confirmation of the dominant role of these currents, we further tested a subset of neurons $(n=4)$ with extended-duration current stimuli designed specifically to evoke slower-activating potassium currents $(>2 s)$, such as sodiumdependent potassium currents. Stimuli were current pulses with a duration of $20 \mathrm{~s}$; the protocol was adapted from Descalzo et al. (2005). We found no significant hyperpolarization activating over a slower timescale (data not shown).

\section{Weak correlation between adaptation and sodium current inactivation}

Slow inactivation of sodium currents can contribute strongly to adaptation during maintained stimulation (Powers et al., 1999; Blair and Bean, 2003; Kim and Rieke, 2003; Miles et al., 2005). In the cortex, long-lasting stimuli can generate a significant amount of slow inactivation, which raises spike thresholds and slows down spikes (Fleidervish et al., 1996). Sodium current availability during sustained spiking can be strongly affected by inactivation, which can therefore impact repetitive firing more than it does individual action potential waveforms (Madeja, 2000). One variable that is a direct function of availability and thus provides a convenient surrogate measure is the voltage threshold for spike firing (Kim and Rieke, 2003). We therefore measured spike thresholds (see Materials and Methods) and found that they differed clearly across high- and low-variance epochs for rectified stimuli: high-variance thresholds were higher (for an example, see right trace in Fig. 2 A). Across neurons, the difference between high- and low-variance threshold at steady-state ranged between 1 and $19 \mathrm{mV}$ (mean \pm SEM of $7.0 \pm 0.5 \mathrm{mV} ; n=59$ ). We found no significant correlation between the magnitude of differences in threshold and the magnitude of the adaptation ratio $(n=58$; $p=0.51$; Spearman's $\rho^{2}=0.0077$ ). This indicates that slow sodium current inactivation did not play a significant role in barrel cortex adaptation, at least within the range of firing fre-

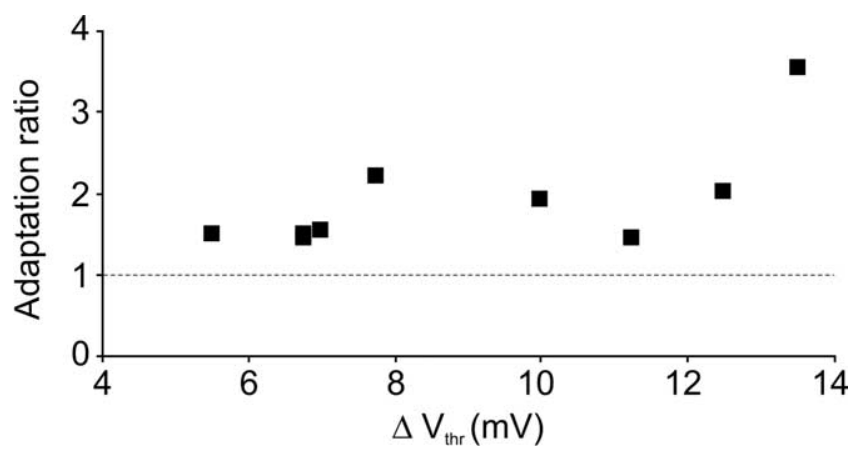

Figure 8. Weak correlation between adaptation and sodium current inactivation. Plot shows data from responses to rectified stimuli in the presence of $\alpha$ me5HT $(n=9)$. The adaptation ratio value of each neuron is plotted against the corresponding difference in spike threshold between high-and low-variance epochs $\left(\Delta V_{\text {thr }}\right)$. Although these data showed a correlation between adaptation ratio and $\Delta V_{\text {thr }}$, overall data using standard ACSF did not.

quencies examined. We did find a correlation between the magnitude of differences in threshold and the magnitude of adaptation for recordings in the presence of $\alpha$ me5HT $(n=9 ; p=0.047$; Pearson's $r^{2}=0.45$ ) (Fig. 8). This suggests that there may be a small effect of inactivation that is occluded when other adaptive mechanisms (namely, the sAHP) are intact.

\section{Discussion}

We have shown that the intrinsic properties of barrel cortex neurons can sustain adaptive gain rescaling that makes responses invariant to the scale of the current stimulus distribution. This form of adaptation adjusts the "operating point" of the neurons (Ringach and Malone, 2007) and is similar phenomenologically and functionally to adaptation to stimulus statistics in vivo (Maravall et al., 2007). The behavior is associated with mechanisms that are widespread across neurons but is highly sensitive to the form of stimulation; the crucial requirement is that neurons receive rectified inputs.

\section{Intrinsic gain regulation}

The gain of the responses of cortical neurons to a specified signal depends on synaptic background noise (Harsch and Robinson, 2000; Chance et al., 2002; Fellous et al., 2003; Mitchell and Silver, 2003; Rauch et al., 2003; Shu et al., 2003). Recently, Higgs et al. found that intrinsic mechanisms regulate how background noise affects the gain of responses to a parametrically varying mean DC signal (Higgs et al., 2006). Neurons can also modulate their sensitivity to fluctuations (i.e., noise) over a range of changes in stimulus mean (i.e., signal) (Arsiero et al., 2007). Our present findings show that intrinsic mechanisms can modulate neuronal gain under changes in the distribution of sensory inputs, when signal and noise are not identified a priori.

\section{Intrinsic mechanisms of adaptation}

Adaptation to rectified stimuli was reduced by blocking calciumdependent currents (Fig. 7C); moreover, the correlation between calcium-dependent sAHP and adaptation ratio was strong (Fig. $7 F$ ). These experiments suggest a link between barrel cortex adaptation to stimulus statistics and the expression of a particular ionic current. A recent study involving simulations of model neurons with a calcium-dependent sAHP predicted that the sAHP may drive adaptation caused by changes in mean but is not activated by pure changes in variance (Garcia-Lazaro et al., 2007). Although the stimulation protocols are not directly comparable, our experimental results show stimulus-specific adaptation cor- 
related with SAHP magnitude and thus appear at least partially consistent with this prediction.

The sAHP did not fully explain adaptation: significant adaptation remained after the sAHP was abolished by $\mathrm{CdCl}_{2}$ (Fig. $7 \mathrm{~B}, C)$, and variations in sAHP magnitude accounted for $32 \%$ of the variance in adaptation ratio value (Fig. $7 F$ ). Recent work shows that, in cortical pyramidal neurons, sAHP magnitude and the gain of responses to DC stimuli are correlated by a similar amount $\left(r^{2}=0.23\right)$ (Higgs et al., 2006).

Other known intrinsic mechanisms covaried weakly, at best, with adaptation. There was no evidence of participation of sodium-dependent potassium currents. Also, spiking thresholds differed in high- and low-variance stimulation periods, a result suggestive of sodium current inactivation; however, differences in threshold across periods did not covary with adaptation magnitude.

Other authors have found no evidence that intrinsic mechanisms participate in adaptation to discrete, repeated whisker displacements (Chung et al., 2002; Katz et al., 2006). Adaptation can depend on the details of the stimuli: intrinsic mechanisms may be activated to different degrees by different forms of stimulation. For example, slowly activating currents may fail to be recruited by sequences of discrete, well separated short whisker displacements.

\section{Association between adaptation and gain rescaling}

Rate adaptation to rectified stimuli over several hundred milliseconds was associated with full gain rescaling, i.e., gain rescaling that compensated for changes in stimulus distribution range (Figs. 2-5). In other systems, adaptation over hundreds of milliseconds can accompany a tonic shift in membrane potential consistent with SAHP currents or slow inactivation of sodium currents, whereas changes in filter shape or in gain depend on faster mechanisms (Fairhall et al., 2001; Baccus and Meister, 2002; Nagel and Doupe, 2006) (for review, see Wark et al., 2007). In fact, gain rescaling can be fast enough to be accounted for by fixed nonlinearities associated with spiking and acting within the timescale of the filter itself rather than true alterations in the stimulusresponse relationship of the neurons (Aguera y Arcas and Fairhall, 2003; Borst et al., 2005; Nagel and Doupe, 2006; Gaudry and Reinagel, 2007). Gain rescaling attributable to generic nonlinear behavior could occur even in neurons expressing negligible rate adaptation, including simplified integrate-and-fire model neurons (Rudd and Brown, 1997; Aguera y Arcas and Fairhall, 2003; Paninski et al., 2003; Borst et al., 2005; Yu et al., 2005; Gaudry and Reinagel, 2007). There is no direct evidence in our data on whether rate adaptation and full gain rescaling had common or separate mechanisms, although partial gain rescaling occurred even when rate adaptation was small or negligible (Figs. $3 A, E$, $5 B, C$ ), consistent with previous results (Paninski et al., 2003). However, we only found full gain rescaling under the same conditions as rate adaptation, namely, under rectified stimuli whose mean and variance switched jointly. Furthermore, neurons lacking full rescaling (counterexamples to generic gain rescaling) are found experimentally in barrel cortex (Maravall et al., 2007) and in the trigeminal ganglion and ventroposterior medial thalamic nucleus (A. Alenda, M. Brambilla, M. Bale, R. S. Petersen, and M. Maravall, unpublished observation). Together, this evidence suggests that full gain rescaling depends nontrivially on stimulation mode and occurs under circumstances that also induce rate adaptation, whereas partial rescaling may be a generic property of spiking neurons.

\section{Stimulation regimen}

Our recordings explored a "noise-dominated regimen" in which fluctuations in current, rather than its mean value, were the main factor driving neurons; spikes were evoked in an irregular, ongoing manner (Maravall et al., 2007). Barrel cortex membrane potential trajectories are highly irregular in both the anesthetized and awake states (Margrie et al., 2002; Petersen et al., 2003; Sachdev et al., 2004; Crochet and Petersen, 2006; Waters and Helmchen, 2006): wakeful periods of active whisking generate small, fast fluctuations in membrane potential that depend on whisker position (Crochet and Petersen, 2006). However, it was beyond the scope of this study to reproduce differences between anesthetized, awake passive and awake, actively exploring states (Crochet and Petersen, 2006).

In the noise-dominated regimen, changes in mean stimulus magnitude did not evoke appreciable changes in steady-state firing rate (Fig. $5 A$ ). This contrasted with the ample dynamic range of cortical neurons when the magnitude of noise is small compared with the range of variations in mean (Higgs et al., 2006). Thus, neuronal input-output functions were strongly dampened by noise, an idea that fits in with known results (Destexhe et al., 2001; Chance et al., 2002; Fellous et al., 2003; Rauch et al., 2003; Shu et al., 2003). However, neurons reliably and precisely reported changes in stimulus mean (Fig. $5 A$ ) as well as in variance. This agrees with suggestions from previous experiments and with predictions from modeling studies (Silberberg et al., 2004; Fourcaud-Trocme and Brunel, 2005).

The stimuli used in this study were far from saturating neurons. This was supported by the lack of change in filter waveforms with switches in stimulus distribution, which agreed with our findings in vivo (Maravall et al., 2007) and with behavior in other systems under stimuli that switch variance with no change in mean (Nagel and Doupe, 2006). This similarity between slice and in vivo findings is consistent with the idea that sensory whisker stimuli rarely saturate cortical neurons. Indeed, in vivo intracellular studies have shown that barrel cortex activity is sparse (Margrie et al., 2002; Petersen et al., 2003; Sachdev et al., 2004; Crochet and Petersen, 2006; Waters and Helmchen, 2006). Because responses in barrel cortex appear to be far from saturation, its avoidance is probably not a critical function of adaptive gain rescaling (Ringach and Malone, 2007).

\section{Functional implications for vibrissa motion encoding}

If adaptive gain rescaling is not needed to avoid saturation, what functions might it have? One possibility is that adaptation underlies normalization of texture encoding. Rodents can modulate the overall amplitude and speed of whisking (for review, see Kleinfeld et al., 2006). During damped whisker motion, cortical neurons represent texture identity by responding to the whisker vibration patterns induced as whiskers sweep across the texture (Arabzadeh et al., 2005, 2006). Ideally, texture perception should be invariant to the details of the whisking motion with which the texture is explored; responses underlying discrimination should carry information specific to the texture. Adaptation could help neurons achieve this by making responses to texture-induced whisker vibrations invariant to the overall scale of the whisking motion, which does not provide texture-specific information (Maravall et al., 2007).

Adaptive gain rescaling is favored by rectified inputs. Rectification is experimentally found in vivo under white noise stimulation and corresponds to symmetric directional selectivity (Arabzadeh et al., 2005; Hasenstaub et al., 2007; Maravall et al., 2007). Rectification of whisker synaptic inputs may be frequency 
dependent, occurring when stimuli contain relatively high frequencies $(\sim 100-200 \mathrm{~Hz})$ (Puccini et al., 2006). Such frequencies are reached by whisker vibrations during texture contact (Arabzadeh et al., 2005) but not during free whisking, which occurs at 5-25 Hz. Because rectification favors gain rescaling, its frequency dependence could also make gain rescaling in downstream neurons frequency dependent. If so, full rescaling would be prominent during texture discrimination, whereas responses during free motion would depend on overall whisking amplitude.

\section{References}

Aguera y Arcas B, Fairhall AL (2003) What causes a neuron to spike? Neural Comput 15:1789-1807.

Aguera y Arcas B, Fairhall AL, Bialek W (2003) Computation in a single neuron: Hodgkin and Huxley revisited. Neural Comput 15:1715-1749.

Ahissar E, Sosnik R, Haidarliu S (2000) Transformation from temporal to rate coding in a somatosensory thalamocortical pathway. Nature 406:302-306.

Ahissar E, Sosnik R, Bagdasarian K, Haidarliu S (2001) Temporal frequency of whisker movement. II. Laminar organization of cortical representations. J Neurophysiol 86:354-367.

Arabzadeh E, Zorzin E, Diamond ME (2005) Neuronal encoding of texture in the whisker sensory pathway. PLoS Biol 3:e17.

Arabzadeh E, Panzeri S, Diamond ME (2006) Deciphering the spike train of a sensory neuron: counts and temporal patterns in the rat whisker pathway. J Neurosci 26:9216-9226.

Arsiero M, Luscher HR, Lundstrom BN, Giugliano M (2007) The impact of input fluctuations on the frequency-current relationships of layer 5 pyramidal neurons in the rat medial prefrontal cortex. J Neurosci 27:3274-3284.

Baccus SA, Meister M (2002) Fast and slow contrast adaptation in retinal circuitry. Neuron 36:909-919.

Bialek W, De Ruyter Van Steveninck RR (2005) Features and dimensions: motion estimation in fly vision. Retrieved January 4, 2008, from http://arxiv.org/abs/q-bio/0505003.

Blair NT, Bean BP (2003) Role of tetrodotoxin-resistant $\mathrm{Na}^{+}$current slow inactivation in adaptation of action potential firing in small-diameter dorsal root ganglion neurons. J Neurosci 23:10338-10350.

Bonin V, Mante V, Carandini M (2006) The statistical computation underlying contrast gain control. J Neurosci 26:6346-6353.

Borst A, Flanagin VL, Sompolinsky H (2005) Adaptation without parameter change: dynamic gain control in motion detection. Proc Natl Acad Sci USA 102:6172-6176.

Brenner N, Bialek W, de Ruyter van Steveninck R (2000) Adaptive rescaling maximizes information transmission. Neuron 26:695-702.

Bryant HL, Segundo JP (1976) Spike initiation by transmembrane current: a white-noise analysis. J Physiol (Lond) 260:279-314.

Carandini M, Ferster D (1997) A tonic hyperpolarization underlying contrast adaptation in cat visual cortex. Science 276:949-952.

Castro-Alamancos MA, Oldford E (2002) Cortical sensory suppression during arousal is due to the activity-dependent depression of thalamocortical synapses. J Physiol (Lond) 541:319-331.

Chance FS, Abbott LF, Reyes AD (2002) Gain modulation from background synaptic input. Neuron 35:773-782.

Chichilnisky EJ (2001) A simple white noise analysis of neuronal light responses. Network 12:199-213.

Chung S, Li X, Nelson SB (2002) Short-term depression at thalamocortical synapses contributes to rapid adaptation of cortical sensory responses in vivo. Neuron 34:437-446.

Crochet S, Petersen CC (2006) Correlating whisker behavior with membrane potential in barrel cortex of awake mice. Nat Neurosci 9:608-610.

de Boer E, Kuyper P (1968) Triggered correlation. IEEE Trans Biomed Eng 15:169-179.

de Ruyter van Steveninck RR, Bialek W (1988) Real-time performance of a movement-sensitive neuron in the blowfly visual system: coding and information transfer in short spike sequences. Proc R Soc Lond B Biol Sci 234:379-414.

Dean I, Harper NS, McAlpine D (2005) Neural population coding of sound level adapts to stimulus statistics. Nat Neurosci 8:1684-1689.

Demb JB (2002) Multiple mechanisms for contrast adaptation in the retina. Neuron 36:781-783.
Derdikman D, Yu C, Haidarliu S, Bagdasarian K, Arieli A, Ahissar E (2006) Layer-specific touch-dependent facilitation and depression in the somatosensory cortex during active whisking. J Neurosci 26:9538-9547.

Descalzo VF, Nowak LG, Brumberg JC, McCormick DA, Sanchez-Vives MV (2005) Slow adaptation in fast-spiking neurons of visual cortex. J Neurophysiol 93:1111-1118.

Destexhe A, Pare D (1999) Impact of network activity on the integrative properties of neocortical pyramidal neurons in vivo. J Neurophysiol 81:1531-1547.

Destexhe A, Rudolph M, Fellous JM, Sejnowski TJ (2001) Fluctuating synaptic conductances recreate in vivo-like activity in neocortical neurons. Neuroscience 107:13-24.

Fairhall AL, Lewen GD, Bialek W, de Ruyter Van Steveninck RR (2001) Efficiency and ambiguity in an adaptive neural code. Nature 412:787-792.

Fellous JM, Rudolph M, Destexhe A, Sejnowski TJ (2003) Synaptic background noise controls the input/output characteristics of single cells in an in vitro model of in vivo activity. Neuroscience 122:811-829.

Fleidervish IA, Friedman A, Gutnick MJ (1996) Slow inactivation of Na+ current and slow cumulative spike adaptation in mouse and guinea-pig neocortical neurones in slices. J Physiol (Lond) 493:83-97.

Fourcaud-Trocme N, Brunel N (2005) Dynamics of the instantaneous firing rate in response to changes in input statistics. J Comput Neurosci 18:311-321.

Garabedian CE, Jones SR, Merzenich MM, Dale A, Moore CI (2003) Bandpass response properties of rat SI neurons. J Neurophysiol 90:1379-1391.

Garcia-Lazaro JA, Ho SS, Nair A, Schnupp JW (2007) Shifting and scaling adaptation to dynamic stimuli in somatosensory cortex. Eur J Neurosci 26:2359-2368.

Gaudry KS, Reinagel P (2007) Benefits of contrast normalization demonstrated in neurons and model cells. J Neurosci 27:8071-8079.

Harsch A, Robinson HP (2000) Postsynaptic variability of firing in rat cortical neurons: the roles of input synchronization and synaptic NMDA receptor conductance. J Neurosci 20:6181-6192.

Hasenstaub A, Sachdev RN, McCormick DA (2007) State changes rapidly modulate cortical neuronal responsiveness. J Neurosci 27:9607-9622.

Higgs MH, Slee SJ, Spain WJ (2006) Diversity of gain modulation by noise in neocortical neurons: regulation by the slow afterhyperpolarization conductance. J Neurosci 26:8787-8799.

Hosoya T, Baccus SA, Meister M (2005) Dynamic predictive coding by the retina. Nature 436:71-77.

Jolivet R, Gerstner W (2004) Predicting spike times of a detailed conductance-based neuron model driven by stochastic spike arrival. J Physiol (Paris) 98:442-451.

Jolivet R, Rauch A, Luscher HR, Gerstner W (2006) Predicting spike timing of neocortical pyramidal neurons by simple threshold models. J Comput Neurosci 21:35-49.

Kaczorowski CC, Disterhoft J, Spruston N (2007) Stability and plasticity of intrinsic membrane properties in hippocampal CA1 pyramidal neurons: effects of internal anions. J Physiol (Lond) 578:799-818.

Katz Y, Heiss JE, Lampl I (2006) Cross-whisker adaptation of neurons in the rat barrel cortex. J Neurosci 26:13363-13372.

Khatri V, Hartings JA, Simons DJ (2004) Adaptation in thalamic barreloid and cortical barrel neurons to periodic whisker deflections varying in frequency and velocity. J Neurophysiol 92:3244-3254.

Kim KJ, Rieke F (2001) Temporal contrast adaptation in the input and output signals of salamander retinal ganglion cells. J Neurosci 21:287-299.

Kim KJ, Rieke F (2003) Slow $\mathrm{Na}^{+}$inactivation and variance adaptation in salamander retinal ganglion cells. J Neurosci 23:1506-1516.

Kleinfeld D, Ahissar E, Diamond ME (2006) Active sensation: insights from the rodent vibrissa sensorimotor system. Curr Opin Neurobiol 16:435-444.

Kvale MN, Schreiner CE (2004) Short-term adaptation of auditory receptive fields to dynamic stimuli. J Neurophysiol 91:604-612.

La Camera G, Rauch A, Luscher HR, Senn W, Fusi S (2004) Minimal models of adapted neuronal response to in vivo-like input currents. Neural Comput 16:2101-2124.

Lee JC, Callaway JC, Foehring RC (2005) Effects of temperature on calcium transients and $\mathrm{Ca}^{2+}$-dependent afterhyperpolarizations in neocortical pyramidal neurons. J Neurophysiol 93:2012-2020.

Madeja M (2000) Do neurons have a reserve of sodium channels for the generation of action potentials? A study on acutely isolated CA1 neurons from the guinea-pig hippocampus. Eur J Neurosci 12:1-7. 
Madison DV, Nicoll RA (1984) Control of the repetitive discharge of rat CA 1 pyramidal neurones in vitro. J Physiol (Lond) 354:319-331.

Mainen ZF, Sejnowski TJ (1995) Reliability of spike timing in neocortical neurons. Science 268:1503-1506.

Maravall M, Stern EA, Svoboda K (2004) Development of intrinsic properties and excitability of layer $2 / 3$ pyramidal neurons during a critical period for sensory maps in rat barrel cortex. J Neurophysiol 92:144-156.

Maravall M, Petersen RS, Fairhall AL, Arabzadeh E, Diamond ME (2007) Shifts in coding properties and maintenance of information transmission during adaptation in barrel cortex. PLoS Biol 5:e19.

Margrie TW, Brecht M, Sakmann B (2002) In vivo, low-resistance, wholecell recordings from neurons in the anaesthetized and awake mammalian brain. Pflügers Arch 444:491-498.

McCormick DA, Connors BW, Lighthall JW, Prince DA (1985) Comparative electrophysiology of pyramidal and sparsely spiny stellate neurons of the neocortex. J Neurophysiol 54:782-806.

Miles GB, Dai Y, Brownstone RM (2005) Mechanisms underlying the early phase of spike frequency adaptation in mouse spinal motoneurones. J Physiol (Lond) 566:519-532.

Mitchell SJ, Silver RA (2003) Shunting inhibition modulates neuronal gain during synaptic excitation. Neuron 38:433-445.

Muller JR, Metha AB, Krauskopf J, Lennie P (1999) Rapid adaptation in visual cortex to the structure of images. Science 285:1405-1408.

Nagel KI, Doupe AJ (2006) Temporal processing and adaptation in the songbird auditory forebrain. Neuron 51:845-859.

Nowak LG, Sanchez-Vives MV, McCormick DA (1997) Influence of low and high frequency inputs on spike timing in visual cortical neurons. Cereb Cortex 7:487-501.

Paninski L (2003) Convergence properties of three spike-triggered analysis techniques. Network 14:437-464.

Paninski L, Lau B, Reyes A (2003) Noise-driven adaptation: in vitro and mathematical analysis. Neurocomputing 52:877-883.

Petersen CC, Hahn TT, Mehta M, Grinvald A, Sakmann B (2003) Interaction of sensory responses with spontaneous depolarization in layer $2 / 3$ barrel cortex. Proc Natl Acad Sci USA 100:13638-13643.

Powers RK, Sawczuk A, Musick JR, Binder MD (1999) Multiple mechanisms of spike-frequency adaptation in motoneurones. J Physiol (Paris) 93:101-114.

Puccini GD, Compte A, Maravall M (2006) Stimulus dependence of barrel cortex directional selectivity. PLoS ONE 1:e137.

Rauch A, La Camera G, Luscher HR, Senn W, Fusi S (2003) Neocortical pyramidal cells respond as integrate-and-fire neurons to in vivo-like input currents. J Neurophysiol 90:1598-1612.

Richardson MJE (2004) Effects of synaptic conductance on the voltage distribution and firing rate of spiking neurons. Physical Rev E 69:051918.

Rieke F (2001) Temporal contrast adaptation in salamander bipolar cells. J Neurosci 21:9445-9454.

Ringach DL, Malone BJ (2007) The operating point of the cortex: neurons as large deviation detectors. J Neurosci 27:7673-7683.

Rudd ME, Brown LG (1997) Noise adaptation in integrate-and fire neurons. Neural Comput 9:1047-1069.

Rust NC, Schwartz O, Movshon JA, Simoncelli EP (2005) Spatiotemporal elements of macaque v1 receptive fields. Neuron 46:945-956.

Sachdev RN, Ebner FF, Wilson CJ (2004) Effect of subthreshold up and down states on the whisker-evoked response in somatosensory cortex. J Neurophysiol 92:3511-3521.

Sanchez-Vives MV, Nowak LG, McCormick DA (2000a) Membrane mechanisms underlying contrast adaptation in cat area 17 in vivo. J Neurosci 20:4267-4285.

Sanchez-Vives MV, Nowak LG, McCormick DA (2000b) Cellular mechanisms of long-lasting adaptation in visual cortical neurons in vitro. J Neurosci 20:4286-4299.

Schwartz O, Pillow JW, Rust NC, Simoncelli EP (2006) Spike-triggered neural characterization. J Vis 6:484-507.
Schwindt PC, Spain WJ, Foehring RC, Chubb MC, Crill WE (1988) Slow conductances in neurons from cat sensorimotor cortex in vitro and their role in slow excitability changes. J Neurophysiol 59:450-467.

Schwindt PC, Spain WJ, Crill WE (1989) Long-lasting reduction of excitability by a sodium-dependent potassium current in cat neocortical neurons. J Neurophysiol 61:233-244.

Shapley R, Enroth-Cugell C, Bonds AB, Kirby A (1972) Gain control in the retina and retinal dynamics. Nature 236:352-353.

Shapley RM, Victor JD (1978) The effect of contrast on the transfer properties of cat retinal ganglion cells. J Physiol (Lond) 285:275-298.

Shapley RM, Victor JD (1979) Nonlinear spatial summation and the contrast gain control of cat retinal ganglion cells. J Physiol (Lond) 290:141-161.

Sharpee TO, Sugihara H, Kurgansky AV, Rebrik SP, Stryker MP, Miller KD (2006) Adaptive filtering enhances information transmission in visual cortex. Nature 439:936-942.

Shu Y, Hasenstaub A, Badoual M, Bal T, McCormick DA (2003) Barrages of synaptic activity control the gain and sensitivity of cortical neurons. J Neurosci 23:10388-10401.

Silberberg G, Bethge M, Markram H, Pawelzik K, Tsodyks M (2004) Dynamics of population rate codes in ensembles of neocortical neurons. J Neurophysiol 91:704-709.

Simons DJ (1978) Response properties of vibrissa units in rat SI somatosensory neocortex. J Neurophysiol 41:798-820.

Slee SJ, Higgs MH, Fairhall AL, Spain WJ (2005) Two-dimensional time coding in the auditory brainstem. J Neurosci 25:9978-9988.

Smirnakis SM, Berry MJ, Warland DK, Bialek W, Meister M (1997) Adaptation of retinal processing to image contrast and spatial scale. Nature 386:69-73.

Tang AC, Bartels AM, Sejnowski TJ (1997) Effects of cholinergic modulation on responses of neocortical neurons to fluctuating input. Cereb Cortex 7:502-509.

Thompson SM, Masukawa LM, Prince DA (1985) Temperature dependence of intrinsic membrane properties and synaptic potentials in hippocampal CA1 neurons in vitro. J Neurosci 5:817-824.

Touryan J, Lau B, Dan Y (2002) Isolation of relevant visual features from random stimuli for cortical complex cells. J Neurosci 22:10811-10818.

Velumian AA, Zhang L, Pennefather P, Carlen PL (1997) Reversible inhibition of IK, IAHP, Ih and ICa currents by internally applied gluconate in rat hippocampal pyramidal neurones. Pflügers Arch 433:343-350.

Villalobos C, Beique JC, Gingrich JA, Andrade R (2005) Serotonergic regulation of calcium-activated potassium currents in rodent prefrontal cortex. Eur J Neurosci 22:1120-1126.

Wainwright MJ (1999) Visual adaptation as optimal information transmission. Vision Res 39:3960-3974.

Wark B, Lundstrom BN, Fairhall A (2007) Sensory adaptation. Curr Opin Neurobiol 17:423-429.

Waters J, Helmchen F (2006) Background synaptic activity is sparse in neocortex. J Neurosci 26:8267-8277.

Webber RM, Stanley GB (2004) Nonlinear encoding of tactile patterns in the barrel cortex. J Neurophysiol 91:2010-2022.

Webber RM, Stanley GB (2006) Transient and steady-state dynamics of cortical adaptation. J Neurophysiol 95:2923-2932.

Wilent WB, Contreras D (2005) Dynamics of excitation and inhibition underlying stimulus selectivity in rat somatosensory cortex. Nat Neurosci 8:1364-1370.

Yu Y, Potetz B, Lee TS (2005) The role of spiking nonlinearity in contrast gain control and information transmission. Vision Res 45:583-592.

Zhang L, Weiner JL, Valiante TA, Velumian AA, Watson PL, Jahromi SS, Schertzer S, Pennefather P, Carlen PL (1994) Whole-cell recording of the $\mathrm{Ca}^{2+}$-dependent slow afterhyperpolarization in hippocampal neurones: effects of internally applied anions. Pflügers Arch 426:247-253.

Zhang ZW, Arsenault D (2005) Gain modulation by serotonin in pyramidal neurones of the rat prefrontal cortex. J Physiol (Lond) 566:379-394. 